\title{
Berliner Geowissenschaftlerinnen an der Friedrich-Wilhelms-Universität von 1906 bis 1945, eine Fallstudie
}

\author{
Barbara A. R. Mohr ${ }^{1}$ \& Annette Vogt $^{2}$
}

Mit 9 Abbildungen und 1 Tabelle

\section{Zusammenfassung}

In dieser Untersuchung werden beispielhaft die Lebenswege und Karrieren von Berliner Geowissenschaftlerinnen im Zeitraum von 1906 bis 1945 nachgezeichnet und analysiert. Ähnlich wie an anderen deutschen bzw. westlichen Universitäten, aber im Gegensatz zu Russland, begann die Tätigkeit von Frauen in den Geowissenschaften spät, und das Fach wurde auch relativ selten gewählt, hauptsächlich wegen der zu geringen Berufschancen. Aber die besondere Situation in Berlin mit mehreren sich ergänzenden Institutionen und dem daraus resultierenden breiten Spektrum an geowissenschaftlichen Disziplinen, sowie ausgezeichneten Professoren, ließ dennoch Raum für eine Ausbildung in diesem Bereich und erlaubte, wenn auch in bescheidenem Maße, eine gewisse Karrieremöglichkeit.

Während der hier untersuchten 40 Jahre haben weniger als 20 Frauen in den Geowissenschaften und benachbarten Gebieten promoviert. Mehrere dieser Frauen blieben in dem von ihnen gewählten Fach weiterhin aktiv und wurden erfolgreich. Zwei Frauen gelang eine akademische Karriere - eine als Universitätsprofessorin, die auch Schülerinnen hatte. Andere arbeiteten an staatlichen Institutionen, wie z. B. dem Geologischen Landesamt. Wenige Frauen blieben nach ihrer Verheiratung beruflich aktiv, wenn auch nicht offiziell angestellt, sondern als Ehefrauen. Andere arbeiteten als „Ersatz“ für die im Kriege stehenden Männer. Einige waren aus persönlichen und politischen Gründen, insbesondere während der NS-Zeit, gezwungen, die Geowissenschaften zu verlassen, konnten aber teilweise auf anderen Gebieten erfolgreich arbeiten.

Schlüsselwörter: Geowissenschaftlerinnen, Berliner Universität, erste Hälfte des 20. Jahrhunderts, Karrieremöglichkeiten

\section{Summary}

Berlin women geoscientists from 1906-1946, a case study

This paper documents the lives and careers of women geoscientists at the Berlin Friedrich-Wilhelms-University from 1906 through 1945. Traditionally, in Germany, women had difficulties to be accepted in geosciences (except for geography/geology teachers), because of strong links between geology and mining, a field dominated clearly by men. In western European countries, as well as in the U.S.A. and Australia, the situation was similar in that women started late and in small numbers to study geology. This was, however, in contrast to Russia and later the Soviet Union where women were relatively early accepted even as university teachers.

The data for this paper were gathered from Berlin University institutions, such as the historical archive and the library of the Palaeontological Institute, and in addition personal contacts were used. Women who had studied either geography, geology/palaeontology, geophysics, mineralogy or botany/palaeobotany are subject of this study. Only those are considered who had strong affiliations to geosciences proper, in all 17 women.

During the first half of the 20th century the Berlin Friedrich-Wilhelms-University, founded in 1810, was one of the most important institutions concerning higher education in Germany, especially for women. The official opening of this university for women students was in 1908, somewhat later than at other German universities. Once admitted, however, the number of dissertations completed by women was relatively high, and, $30 \%$ of all habilitations (advanced degree which allows teaching at universities) in Germany and 50\% of all habilitations in the natural sciences were accomplished at Berlin between 1918/19 to 1932 .

The geosciences were, together with medicine, chemistry, physics, botany and zoology, very strong scientifical and in teaching. Geoscientists of international reputation worked at large institutions, affiliated or being part of the University, such as the Prussian (later German) Geological Survey, the Institute of Geology and Palaeontology at the Museum of Natural History or the Institute and Museum of Oceanography, and were the advisers and reviewers of women Diploma and PhD students.

\section{Women's careers}

The first woman graduate in geography (1906), Helene Wiszwianski from Switzerland (born in 1881), was still not regularly enrolled, but studied with "special permission" at the Institute and Museum of Oceanography.

\footnotetext{
1 Museum für Naturkunde der Humboldt-Universität zu Berlin, Invalidenstr. 43, 10115 Berlin, Germany. E-mail: barbara.mohr@rz.hu-berlin.de

${ }^{2}$ Max-Planck-Institut für Wissenschaftsgeschichte, Wilhelmstr. 44, 10117 Berlin, Germany.

E-mail: vogt@mpiwg-berlin.mpg.de

Received February 2003, accepted July 2003
} 
In 1913 Antonie Täuber was the first to finish successfully her PhD thesis in geology. She got married to the Assistant Professor H. Stremme at the Geological/Palaeontological Institute, who later became the Director of the Mineralogical/Geological Institute at Danzig (1918-1939 an independent State). Antonie, called Toni, substituted for her husband in his position during the two world wars. The family fled from Danzig when the city became part of Poland, to Berlin (East) where Prof. Stremme was offered the directorship of an Institution dedicated to Soil Sciences, while Toni worked on maps for the Berlin magistrate.

Charlotte (Lotte) Möller (PhD 1925) was besides Gertrud Kobe (PhD 1934) one of the few women who had a successful university career, first at the Berlin university where she became assistant professor. In 1945 she lost her job, because of her affiliation to NS-organisations, but later worked at the Göttingen university.

In 1928, Hertha Sieverts-Doreck completed her thesis on a palaeozoological theme, more precisely on crinoids with F. J. Pompeckj. She married, had two daughters and did not have a paid position, but she published about 70 papers on crinoids and was a to contributor to the "Treatise on Invertebrate Paleontology".

During the first years of the NS-regime, the situation for women deterioriated dramatically. According to the NS-doctrine, only $10 \%$ of all students could be women which meant that only academically very gifted girls with excellent high school degrees were allowed into the university. In addition, the pressure for people by the NS-regime who were considered to be "politically" or "racially" not "correct", grew enormously since 1933.

Gertrud Denner-Bobek graduated in 1928 in geography. She had married a chemist. Dr. Felix Bobek, who was member of the German Communist Party where Gertrud also became deeply involved. In 1937, Felix was killed by the NS-Government, and Gertrud had to flee with her two daughters to the Soviet Union. After 1945, she remigrated to the Russian occupied part of Germany and became a political figure in the GDR (German Democratic Republic).

Hilde Wohlgemuth, member of a jewish merchant family in western Germany, graduated in 1935 in geography/geology. She was able to save her life by emigrating to Sweden where she published several books and bibliographies on Jewish history.

As one of the few women with excellent high school grades, Marie-Luise Teichmüller, called Marlies, was accepted to study geology during the early NS-period. She studied abroad in Pittsburg. U.S.A., in 1938 with a stipend in order to learn various methods in the field of coal petrology. She successfully finished her thesis in 1941 and after World War II had a great career as one of the directors at the Geological Survey of North Rhine-Westfalia at Krefeld where she worked scientifically and published intensely (more than 100 papers) until her death.

\section{Results}

The general attitude at German universities during the first half of the 20th century was conservative, and women, especially in Berlin, had the right of regular enrolment only late (1908/9). In contrast to other sciences such as medicine and chemistry, the entry of women into geosciences was even more delayed and rare. However, the special role of the Berlin University as the leading German university in sciences with a large variety of subfields in geosciences at several independent institutions, gave women a somewhat better chance to find supervisors for their $\mathrm{PhD}$ studies, as well as (very rarely) the chance to stay in academia than at many of the smaller universities

The post-PhD careers of several women, mentioned in this paper, is unknown (8). Of those whose curriculum vitae are known, most worked professionally (6-7). Many of the women worked in various professions after they had gained their $\mathrm{PhD}$, such as academic teachers at the university (2), in positions of academies and geological surveys (3), and school teachers (1). Some of them stayed active in the field, published on scientific themes or did work in geological institutions, but were not officially employed (1-2). Some had to leave the field completely either for private or political reasons, but were successful in other fields.

Key words: Women geoscientists, Berlin University, first half of the 20th Century, professional careers.

\section{Einleitung}

In vielen akademischen Berufen, wie in der Medizin, sind Frauen seit etwa 100 Jahren außerordentlich aktiv und erfolgreich. In den Geowissenschaften ist und war dies jedoch nur teilweise der Fall. Zwar waren in den 90er Jahren des 20 . Jh. ca. $30-40 \%$ der Studierenden weiblich, die Zahl der Professorinnen innerhalb der Geologie/Paläontologie liegt aber bei weniger als $3 \%$ (Kölbl-Ebert 2001), was neben allgemeinen gesellschaftlichen, z. B. wenig Kinderbetreuung nach der Schule, auch fachspezifische Ursachen hatte und weiterhin hat (Mohr \& Vogt 2001). Die deutliche Unterrepräsentation von Frauen in den Geowissenschaften, ihre Schwierigkeiten innerhalb dieses Bereichs professionell tätig zu sein, aber auch vereinzelte „success stories” sol- len anhand dieser Fallstudie, die die Situation an der Berliner Universität vor dem 2. Weltkrieg untersucht, dargestellt werden.

Die geringe Anzahl von Studentinnen und das späte Einsetzen von geowissenschaftlicher Forschung durch Frauen ist jedoch nicht nur auf Deutschland beschränkt. In anderen europäischen, besonders in den angelsächsischen Ländern, war die Situation ähnlich. Zwar war es in der Schweiz möglich, bereits früh ein GeologieStudium zu absolvieren, wie das Beispiel Marie Jerosch (1877-1952) in Zürich zeigt. Sie arbeitete später mit ihrem Mann, einem Botaniker, im Bereich Geobotanik der Alpen. Sie war aber ein Einzelfall.

Für die angelsächsischen Länder gelten ähnliche Verhältnisse. Dort waren die meisten Geologinnen zunächst in Gemeinschaft mit ihren 
Ehemännern tätig, z. B. Charlotte Murchison (1788-1869) (Kölbl-Ebert 1997). In den U.S.A. waren und sind die Zustände im Prinzip vergleichbar, allerdings gab es bereits früher die Möglichkeit ein Studium zu absolvieren. Emilée Holmes z. B. konnte bereits 1888 ihre Dissertation abschließen; Carrie A. Harbour wurde bereits 1893 Kustos an der State University of Nebraska, später auch Professorin (Schwarzer \& Crawford 1977). Dennoch waren lange Frauen in Ämtern eine kleine Minderheit und noch 1992 waren lediglich $19 \%$ aller Doktoranden in den Geowissenschaften Frauen, gegenüber $42 \%$ an Studienanfängern (Suiter 1992). 1999 waren etwa 9-10\% der Universitätswissenschaftler Frauen (Wolfe 1999). Auch in Australien waren Frauen in den ersten 120 Jahren meist als Illustratoren und Sammlerinnen tätig. Erst Mitte des 20. Jh. erhielten Frauen reguläre Anstellungen. Ida Brown z. B., die ursprünglich Petrologie studiert hatte, wurde als eine der ersten Frauen ,senior lecturer" an der Universität in Sydney während der 40er-50er Jahre (Turner 1994).

Anders war es dagegen im zaristischen und später kommunistischen Rußland. Dem russischen Akademiemitglied Dmitrij Vasil'evic Nalivkin fiel bei seinen „West-Reisen“ auf, daß nirgendwo so viele Geologinnen tätig waren wie in der Sowjetunion. Daher untersuchte er die Vorgeschichte und suchte die Spuren der 19 „Pionierinnen" der russisch-sowjetischen Geologie (Nalivkin 1979). Er stellte die ersten zwei Generationen von Geologinnen vor, von denen 15 einen akademischen Grad erwarben und 4 als Professorin tätig waren. Die Vertreterinnen der ersten Generation (zwischen 1854 und 1877 geboren) konnten nur extern das nötige Wissen erwerben und waren meist mit ihrem Ehemann tätig. Die Vertreterinnen der zweiten Generation (zwischen 1887 und 1901 geboren) absolvierten die höheren Frauenkurse, vor allem in St. Petersburg und Moskau, wo ihnen Universitätsprofessoren solide Kenntnisse vermittelten und sie zum Teil als Assistentinnen an der Universität anstellten, obwohl die Universitäten im zaristischen Rußland bis 1917 den Frauen verschlossen blieben (vgl. auch Reck \& Kaufer 1997). Nach 1917 waren sie an der wachsenden Zahl von Expeditionen, besonders nach Sibirien, beteiligt, und einige arbeiteten im Trust der Erdöl- und Erdgasförderung. Die so ausgebildeten Frauen hatten im sowjetischen System eine gute Chance, in Akademie-Instituten und Universitäten vergleichsweise hohe Posten einzunehmen und hunderte Geologen auszubilden.

\section{Methoden}

Die Studie basiert auf Statistiken über Naturwissenschaftlerinnen (Tabelle 1) und detaillierten Daten zu 17 Geowissenschaftlerinnen (s. Appendix) an der Friedrich-Wilhelms-Universität (FWU) zu Berlin und umfasst bereits verstorbene bzw. pensionierte Frauen. Zusätzlich zu publiziertem Material wurden Internet- und persönliche Daten verwendet, die uns teils in Interviews, teils in Briefen mitgeteilt wurden. Dennoch ist das Datenset unvollständig, da bei manchen der bereits verstorbenen Frauen nur auf offizielle schriftliche Zeugnisse zurückgegriffen werden konnte, die naturgemäß wenig über persönliche Lebensumstände Auskunft geben. Dies gilt auch für die wichtige Fragestellung zum Verhältnis zwischen akademischem Betreuer und Promovendin. Lediglich von zwei der hier erwähnten Frauen können wir anhand der Quellenlage Aussagen machen. Als schriftliche Quellen standen uns vor allem das Archiv der Friedrich-WilhelmsUniversität (später Humboldt-Universität) und die Bibliothek des Paläontologischen Instituts (bis $1968 \mathrm{Geologisch} / \mathrm{Pa}$ läontologisches Institut) zur Verfügung. Eine erste Kompilation bzw. Auswertung der in Frage kommenden Daten findet sich im „Findbuch (Index-Book)“ (Vogt 1997).

Berücksichtigt wurden in dieser Studie jene Frauen, die zu geologisch-paläontologischen und petrologischen Themen, zur Geophysik oder zu Themen der physischen Geographie promoviert bzw. gearbeitet haben. Dies war an verschiedenen Instituten, bzw. an der Universität zugeordneten Institutionen möglich, z. B. am Museum für Naturkunde (Mineralogie, Geologie/Paläontologie), und dem Museum für Meereskunde (Geographie).

Tabelle 1

Liste der Promotionen in den Naturwissenschaften von Frauen 1899-1945, an der Friedrich-Wilhelms-Universität zu Berlin.

\begin{tabular}{|c|c|c|c|c|c|c|c|c|c|}
\hline Fachgebiet & $\begin{array}{l}1899- \\
1908\end{array}$ & $\begin{array}{l}1909- \\
1918\end{array}$ & $\begin{array}{l}1919- \\
1932\end{array}$ & $\begin{array}{l}1933- \\
1935\end{array}$ & Summe & $\begin{array}{l}1936- \\
1939\end{array}$ & $\begin{array}{l}1940- \\
1945\end{array}$ & Summe & $\begin{array}{l}\text { Gesamt- } \\
\text { summe }\end{array}$ \\
\hline Chemie & 2 & 27 & 99 & 19 & 147 & 14 & 14 & 28 & 175 \\
\hline Physik & 1 & 6 & 22 & 11 & 39 & 3 & 4 & 7 & 46 \\
\hline Mathematik & & & 5 & 2 & 7 & & 4 & 4 & 11 \\
\hline Astronomie & & & 2 & & 2 & & & & 2 \\
\hline Meteorologie & & & 1 & 1 & 2 & 4 & 2 & 6 & 8 \\
\hline $\begin{array}{l}\text { Zool., Bot., Anthr., } \\
\text { Fischereiwiss. }\end{array}$ & 1 & 8 & 26 & 21 & 56 & 17 & 19 & 36 & 92 \\
\hline Psychologie & & & & & & & 1 & 1 & 1 \\
\hline Geophysik & & & & 1 & 1 & 1 & 1 & 2 & 3 \\
\hline Geologie & & 1 & 1 & & 2 & & 2 & 2 & 4 \\
\hline Mineralogie & & 3 & & & 3 & & & & 3 \\
\hline Geographie/Naturwiss. & 1 & 1 & 2 & 1 & 5 & 2 & 2 & 4 & 9 \\
\hline
\end{tabular}


Zwischen 1906 und 1945 haben insgesamt 21 Frauen mit einem Thema zur Geographie (als Hauptfach) an der FWU promoviert (Vogt 1997). Darunter waren sowohl Themen zur physischen, als auch zur politischen Geographie. Bei der Untersuchung der Geowissenschaftlerinnen im Berliner Raum haben wir bewusst jene Dissertationen von Frauen ausgeklammert, die Themen zur Sozial- bzw. Historischen Geographie behandelten. Letztere wurden wegen der Vergleichbarkeit mit den Geologinnen nicht berücksichtigt. hauptsächlich weil die Berufschancen wesentlich andere waren.

Wir haben dieser Publikation, soweit zugänglich, Photos der hier erwähnten Frauen beigegeben. Obwohl prinzipiell wünschenswert, wurde auf Photos der Betreuer verzichtet, weil sie in den entsprechenden Nachrufen zu finden sind (s. Lit.) und dies den Rahmen dieser Studie sprengen würde.

\section{Frauen in den Geowissenschaften in Deutschland vor 1945}

Generell war die Zahl der Geologiestudentinnen in den mittel- und westeuropäischen Ländern klein (Kölbl-Ebert 2001). Der Grund hierfür war, dass die Berufschancen für Geologinnen und Astronominnen (Vogt 2000b), im Gegensatz zu Physikerinnen und Chemikerinnen (Vogt 2000a), geringer waren, außer im LehrerinnenBeruf.

Bereits früher vermuteten wir, dass neben der damals physisch anstrengenden Gelände- und Kartierarbeit, die enge Verknüpfung zum Bergbau, insbesondere zum Kohlenbergbau, in dem Frauen nur in Ausnahmefällen eine Chance hatten, eine Rolle spielten (Mohr \& Vogt 2001). Betrachten wir die Mitgliederverzeichnisse der Deutschen Geologischen Gesellschaft, wird die ursprünglich kaum zu trennende Einheit von Geologie und Bergbau deutlich. 1909/1910 gab es ca. 600 individuelle Mitglieder, von denen etwa 100 , also ein Sechstel, einen Bergbauberuf (Grubendirektor, Bergrat, Markscheider, etc.) angaben. 1925 waren von den dort angeführten knapp 130 institutionellen Mitgliedern etwa die Hälfte (64) unmittelbar zum Bergbau zugehörig, z. B. Bergämter, Grubengewerkschaften.

Vor 1945 studierten also nur ganz wenige Frauen in Deutschland Geologie im Hauptfach. Dies spiegelt sich auch in den Mitgliederverzeichnissen der Geologischen Vereinigungen wider. Noch 1909, dem Jahr der offiziellen Zulassung von Frauen an die Berliner Universität bzw. in Preußen, war keine Frau im Mitgliederverzeichnis der Deutschen Geologischen Gesellschaft vermerkt. Selbst bis in die 20er Jahre blieb die Zahl der weiblichen Mitglieder in geowissenschaftlichen Vereinigungen sehr klein: 1924 hatte die Deutsche Geologische Gesell- schaft ca. 900 individuelle Mitglieder, aber nur 10 Frauen, was etwa 1\% entsprach (Mohr \& Vogt 2001). Bei anderen geologischen Vereinigungen ergibt sich ein ähnliches Bild. Erst 1919 trat die erste geologisch ausgebildete Frau dem Oberrheinischen Geologischen Verein mit Sitz in Freiburg bei (Villinger 2001). Auch in Unterdisziplinen wie der Paläontologie, die Frauen traditionell mehr ansprachen, war die Zahl begrenzt. 1926 hatte die Deutsche Paläontologische Gesellschaft sechs weibliche Mitglieder bei mehr als 200 männlichen Subskribenten, etwa $3 \%$. Jedoch waren darunter wenige Frauen, die aktiv im Bereich Paläontologie wirkten. Es sei hier Emma Richter (1888-1956), eine Spezialistin für Trilobiten (Dreilappkrebse) und Kustodin an der geologischen Sektion am Senckenberg Museum/Frankfurt genannt. Tilly Edinger (1897-1967), arbeitete in der gleichen Institution bis 1938 als Spezialistin für fossile Gehirne, als sie, bedingt durch die Nazi-Herrschaft, gezwungen wurde, Deutschland $\mathrm{zu}$ verlassen (Kohring 1997).

Ab Mitte der 20er Jahre stieg die Zahl der Geologie-Studentinnen leicht an, denn Geologie konnte im Zuge eines naturwissenschaftlichen Studiums als Nebenfach für das Lehramt/Gymnasium gewählt werden, da Geologie teilweise in naturwissenschaftlich ausgerichteten Oberschulen gelehrt wurde. Das Mitgliederverzeichnis der Geologischen Gesellschaft von 1925 listet 14 Frauen auf, von denen 7 einen Lehrberuf ausübten (1 Lehrerin, 6 Studienrätinnen).

Der Lehrerinnenberuf galt traditionell als frühzeitig anerkannter Beruf für Frauen, der ein Studium gewissermaßen sozial ,rechtfertigte". Die Zulassung von Frauen zu den Universitäten hing unmittelbar mit der Reform des Mädchen-Schulwesens, d.h. der Einrichtung gymnasialer Stufen auch für Mädchen, zusammen. Bereits 1913 galt der Lehrerinnenberuf in beinahe allen naturwissenschaftlichen Fächern als aussichtsreich und diente der Begründung für die Zunahme der Zahl studierender Frauen an den Universitäten (Soden 1913). Die günstigen Berufschancen für Lehrerinnen galten bis Ende der 20er Jahre, als erstmals ein Überangebot existierte. Mit der Abschaffung des naturwissenschaftlichen Abiturs für Mädchen durch die Nationalsozialisten 1938 und der Einführung eines Sprach- (mit nur einer Fremdsprache als Pflichtfach) und Hauswirtschaftzweiges, verschlechterten sich die Chancen für Studentinnen und Lehrerinnen gleichermaßen (Wegel 1993). 


\section{Universitäre Verhältnisse in Deutschland}

Institutionen, die weibliche Promovendinnen offensichtlich schon früh förderten, waren insbesondere die Universitäten im liberalen Südwesten. Eine besondere Rolle spielte das Geologische Institut der Universität Heidelberg. Von 1919 bis 1935 haben insgesamt 9 Frauen unter der Leitung von Wilhelm Salomon Calvi (1868, Berlin - 1941, Ankara; Teichert 1986) studiert bzw. promoviert (Villinger 2000). Von 1925 bis 1935 war dort Ilse Voelcker-Plewe als Assistentin tätig (Rothe 1997).

Auch in Freiburg/Breisgau studierten bereits früh Frauen, die später beruflich erfolgreich waren. Emmy Mercedes Todtmann (1888-1973) begann 1918 als eine der ersten Geologie-Studentinnen Deutschlands dort ihr Studium, das sie in ihrer Heimatstadt Hamburg 1923 beendete und wo sie bis 1947 am Geologischen Landesamt tätig war. Elisabeth Schmidt (1912-1994) beendete 1937 ihr Studium in Freiburg mit einem quartärpaläontologischen Thema, arbeitete danach als Assistentin an den Instituten für Vorgeschichte in Bonn, Köln und Freiburg, wo sie 1949 habilitierte. 1972 wurde sie an den Lehrstuhl für Urgeschichte in Basel berufen (Villinger 2001).

Auch in Frankfurt/M. muss ein offeneres Klima geherrscht haben. Neben den dort tätigen E. Richter und T. Edinger hatte auch Martha Wolf ihre geologische Dissertation unter Rudolf Richter abgeschlossen (Wolf 1930). Da Frankfurt/M. eine neu gegründete Universität (1918) war, könnte der vorurteilsfreiere Umgang mit Frauen an der Struktur der neuen Universität gelegen haben (vgl. Hammerstein 1989).

Die Berliner Friedrich-Wilhelms-Universität bildete zusammen mit mehreren assoziierten Institutionen in der ersten Hälfte des 20. Jahrhunderts eine der wichtigsten akademischen Ausbildungsstätten in Deutschland, auch und besonders für Frauen (s. o.).

Nach der offiziellen Zulassungserlaubnis zum Wintersemester 1908/09 nahm die Zahl der Studentinnen in Berlin rasch zu. Von 6623 eingeschriebenen Hörern waren 1908 bereits 414 Frauen, und 1913 erreichten Frauen einen Anteil von fast $10 \%$, 941 von 10106 eingeschriebenen Studenten (Reinsch \& Lehnert 1996; Lehnert 1998). Die Zahl der abgeschlossenen Dissertationen von Frauen war im Vergleich zu allen anderen deutschen Universitäten hoch. Außerdem wurden fast $30 \%$ aller Habilitationen von Frauen an deutschen Universitäten zwischen 1918/1919 und 1932 an der Berliner Universität abgeschlossen. In Berlin fanden $50 \%$ aller naturwissenschaftlichen Habilitationen, aber nur 19\% der geisteswissenschaftlichen und $18 \%$ der medizinischen statt (Vogt 1997 und 1999).

Das Frauenstudium in den Geowissenschaften setzte jedoch im Verhältnis zu anderen Disziplinen in Berlin erst relativ spät und zögerlich ein (Tabelle 1). Auch in den nächsten 30 bis 40 Jahren wählten vergleichsweise wenig Frauen eine berufliche Karriere in diesem Bereich. Bis 1945 zählten wir weniger als 20 Frauen. Es promovierten 3 Frauen zur Geologie/Paläontologie, 2 zur Paläobotanik, 3 zur Mineralogie, wobei jedoch 2 der 3 Frauen chemische Fragestellungen behandelten, nur 2 zur Geophysik sowie 9 Frauen zur physischen Geographie, also insgesamt 17 zu den Geowissenschaften. Gegenüber anderen deutschen Universitäten ist dies eine relativ hohe Zahl. Deshalb besitzen Studien, die sich mit der Frauengeschichte dieser Universität befassen, für deutsche Verhältnisse einen exemplarischen Charakter.

Erst während der 50er Jahre änderte sich dies in Ostdeutschland (DDR seit 1949, einschließlich Berlin Ost), als Frauen eine wichtige Rolle im System der Energieversorgung einnehmen sollten. Dagegen blieben im Westen (BRD seit 1949, einschließlich Berlin-West) Dissertationen bzw. berufliche Tätigkeiten in den Geowissenschaften bis in die $80 \mathrm{er}$ bis $90 \mathrm{er}$ Jahre des 20 . Jh. für Frauen eher eine Ausnahme (Mohr \& Vogt 2001).

\section{Geowissenschaften an der Berliner Universität}

Die Berliner Friedrich-Wilhelms-Universität, gegründet 1810, bildete zusammen mit mehreren assoziierten Institutionen in der ersten Hälfte des 20. Jahrhunderts eine der wichtigsten akademischen Ausbildungsstätten Deutschlands. Neben den damals wissenschaftlich herausragenden Fachbereichen wie Medizin, Chemie, Physik, Botanik und Zoologie nahmen die Geowissenschaften einen wichtigen Platz im Gesamtgefüge der Universität ein. Hier lehrten herausragende Professoren, anerkannte Vertreter ihres Faches, die außerdem alle als Gutachter an Promotionen von Frauen beteiligt waren. Ein integraler Teil dieser Einrichtung bildeten die Geowissenschaften, die im Berliner Raum eine lange Tradition besitzen, die weit vor die Gründungszeit der Universität 1810 zurückreicht. Im Laufe der folgenden Jahrzehnte ergab sich in Berlin eine günstige Konstellation im Bereich der Erdwissen- 


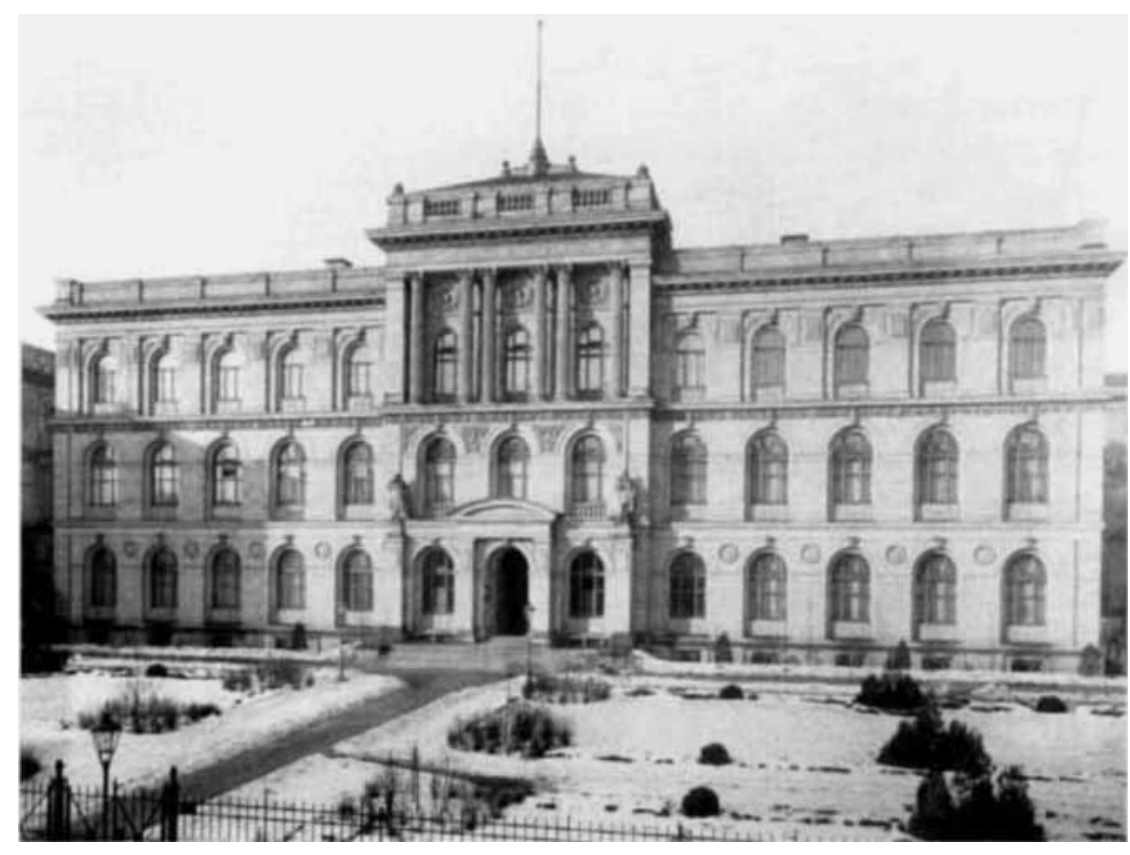

Abb. 1. Vorderansicht des Museums für Naturkunde der Friedrich-Wilhelms-Universität zu Berlin um 1893 (Zool. Mus. B III/1246). schaften, die praktische Belange. wie z. B. Kartierung und Bergwesen, mit dem akademischen Bereich der Forschung und Lehre verknüpfte. Dies gestaltete sich durch die enge institutionelle und personelle Verflechtung des Preussischen Geologischen Landesamtes und des Geologisch/ Paläontologischen Instituts an der Friedrich-Wilhelms-Universität, die bis in die Mitte des 20. Jh. bestand. $\mathrm{Ab} 1889$ waren beide Institutionen auch räumlich eng in den Gebäudekomplexen der Invalidenstr. 43 (Museum für Naturkunde. Abb. 1) und Nr. 44 (Preußische geologische Landesanstalt, später Reichsanstalt für Bodenforschung) miteinander verbunden. In den Jahrzehnten zwischen der Gründung des Deutschen Reiches 1871 und 1910, als die ersten Geowissenschaftlerinnen studierten, erlebte die Universität einen ungeheuren Aufschwung und der Lehrkörper wuchs von 180 auf 508 Dozenten (vgl. Lenz, 1910, Asen 1955). Ab 1900 bzw. 1906 wurde daneben das Institut und Museum für Meereskunde eröffnet, das in der Georgenstrasse einen repräsentiven Bau (Abb. 2) mit Studieneinrichtungen besaß (Bibliothek, Abb. 3, und Kartensaal), aber während des 2. Weltkriegs in Schutt und Asche fiel, und auch nicht mehr aufgebaut wurde.

Das Geologisch/Paläontologische Institut, angesiedelt am Museum für Naturkunde, stand in der Amtszeit von Wilhelm Karl v. Branca (1844-1928) in seiner Blüte (1899-1917). Großangelegte Ausgrabungen in Deutsch-Ostafrika (heute Tansania) führten zu spektakulären Dinosaurierfunden, die bis heute die Publikumsmagneten der öffentlichen Ausstellung sind. W. K. v. Branca hielt Vorlesungen zur allgemei- nen Geologie und Paläontologie und war einer der wichtigsten Gutachter bei Promotionen, so auch bei Antonie Täuber, Dorothea Mesch und Elfriede Gerwien. Ihm stand 1903-1908 als Assistent Privatdozent Hermann Stremme (1879-1961) (Fossile Säugetiere, paläontologisch-osteologische Übungen, Bodenkunde), später Ehemann von A. Täuber (s. unten) zur Seite. Der international anerkannte Paläobotaniker an der Preussischen Geologischen Landesanstalt, Henry Potonié (1857-1913), las ebenfalls an der Universität.

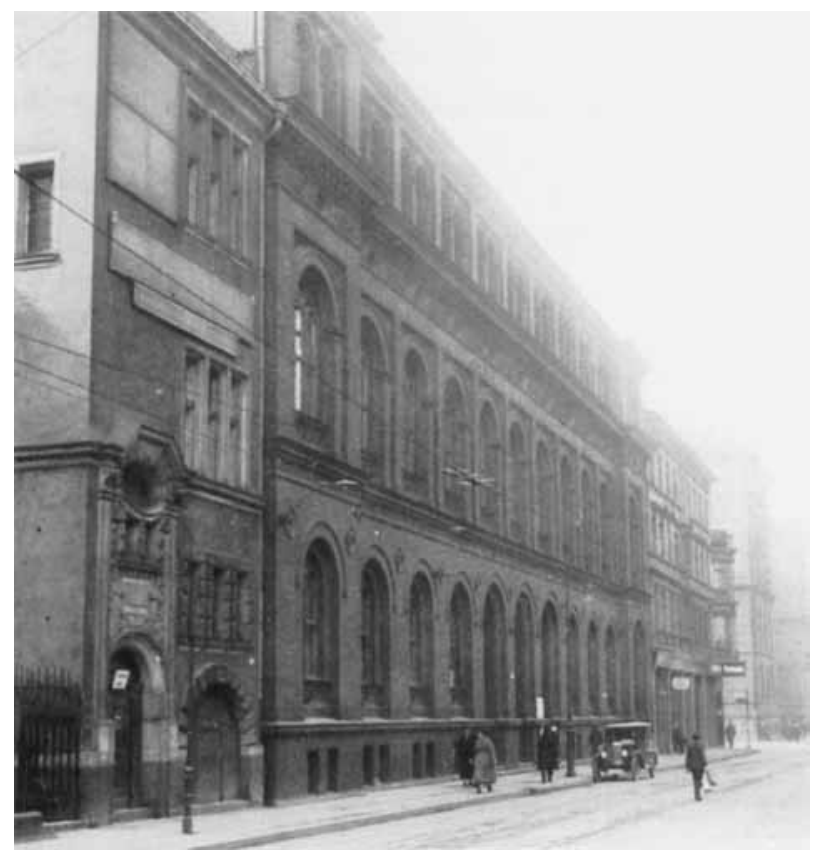

Abb. 2. Institut und Museum für Meereskunde Georgenstr. 34-36 (um 1920?) (aus: Katalog Museum Verkehr und Technik: Aufgetaucht. Das Museum für Meereskunde 1996, S. 6). 


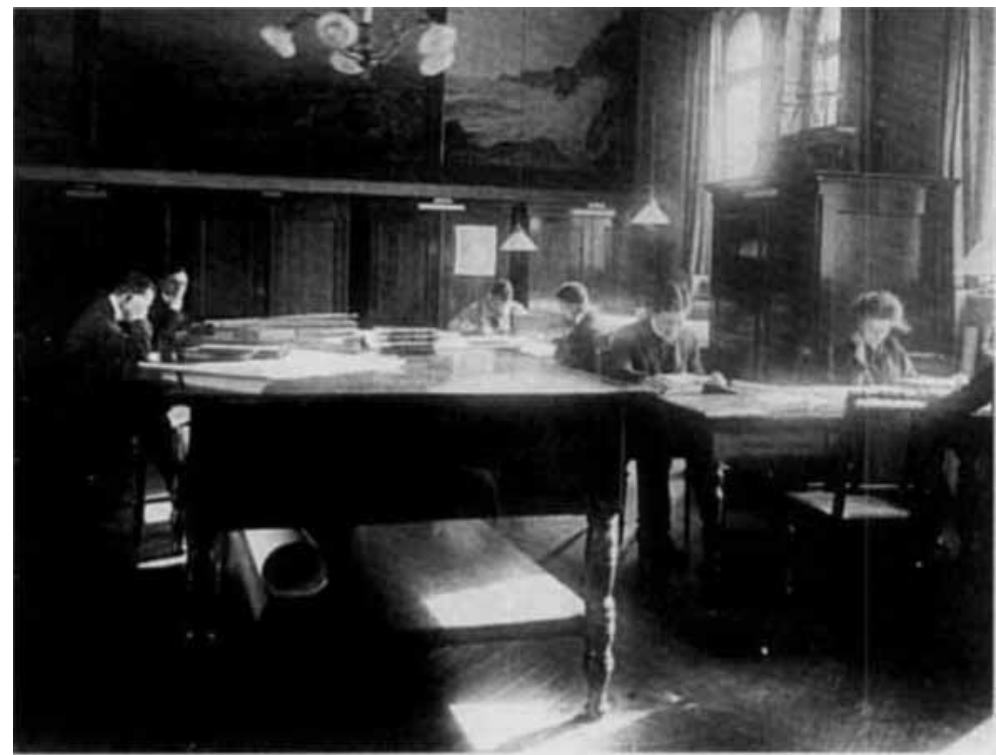

Abb. 3. Lesesaal des Instituts und Museums für Meereskunde (vor 1918?) (aus: Katalog Museum Verkehr und Technik: Aufgetaucht. Das Museum für Meereskunde 1996, S. 57).
1917 übernahm Josef Felix Pompeckj (18671930) die Leitung des Geologischen Institutes (Hennig 1930). Im selben Jahr promovierte er noch von seiner Tübinger Zeit her Th. Hoyermann, später verheiratet mit P. Hülsenbeck, ebenfalls Schüler Pompeckj's, mit einem paläozoologischen Thema (Hoyermann 1917). In Berlin unterrichtete er vor allem Paläontologie und Evolutionslehre, aber auch Geologie. Eine seiner Schülerinnen war Hertha Sieverts-Doreck, die 1928 promovierte.

Über die Eiszeit trug Friedrich Solger (18771965) vor (Heck 1966). Er war 1936 als Zweitgutachter bei der Promotion von Irma Schaberow beteiligt.

Walther Gothan (1879-1954), von der PreuBischen geologischen Landesanstalt, hatte als Nachfolger von H. Potonié die Lehre in der Paläobotanik übernommen. Sein Verhältnis zu seinen beiden Schülerinnen (Ferdinande Michael und Ingeborg Böwing) war herzlich (s. unten).

In den 30er Jahren, unter der Leitung von Hans Stille (1876-1966), einem der einflussreichsten Geologen Deutschlands zu dieser Zeit wurde der Unterricht stärker praxisorientiert durchgeführt. In einer Universitäts-Chronik aus dem Jahr 1935/36 wurde berichtet, dass an geologischen Geländekursen und Exkursionen im WS 1935/36 insgesamt 37 Männer und 7 Frauen teilnahmen.

Erich Stach (1896-1987) las Kohlenpetrologie (Teichmüller 1989) und fasste seine langjährige Erfahrung in einem Lehrbuch zur Kohlenpetrographie zusammen (Stach 1935).

Am Institut für Mineralogie, ebenfalls am Museum für Naturkunde angesiedelt, lehrte Theodor Liebisch (1852-1922) insbesondere sein Hauptarbeitsgebiet, die physikalisch-chemische Mineralogie. Er promovierte von 1913-15 drei Frauen, zwei davon jedoch nicht im Bereich Geowissenschaften, sondern in der Chemie (Käthe Sudhaus, 1913 und Ilse Zoch, 1915). Albert Joseph Maria Defant (1884-1974) war seit 1927 Professor für Geophysik. Später war auch Lotte Möller (s. unten) als Betreuerin bzw. Gutachterin von Frauen tätig (Erna Hein, Barbara Wallstabe). Im Bereich Meteorologie leitete Heinrich von Ficker (1881-1957) die Vorlesungen und Prüfungen.

Für die Geowissenschaftlerinnen waren jedoch vor allem die Lehrer im Bereich Geographie von Bedeutung. Albrecht Penck (1858-1945) am Institut für Meereskunde lehrte Allgemeine Geographie. Alfred Merz (1880-1925) wurde 1914 ao. Professor, später 1921 ordentlicher Professor, ab 1923 Direktor des Meereskunde-Instituts. Aufgrund seines frühen Todes während einer zweijährigen Forschungsfahrt in den Südatlantik (Katalog MVT 1996) war er nur einmal als Betreuer bei der Dissertation von Lotte Möller beteiligt. Das Geographische Institut leitete $a b$ 1923 Norbert Krebs (1876-1947), der bei mehreren Promotionen von Frauen sowohl Erst- als auch Zweitgutachter war (Hilde Wohlgemuth, Kläre Masuch, I. Schaberow, B. Wallstabe, E. Hein, Ruth Rabien).

Alle hier genannten zählten zu anerkannten Vertretern ihres Faches und waren als Betreuer und Gutachter bei Promotionen von zahlreichen Studenten beteiligt. Ihre Haltung gegenüber Frauen ist im allgemeinen nicht bekannt. Bei N. Krebs können wir vermuten, dass er auf Grund der Vielzahl von Frauen, die er promovierte, keine Vorurteile hatte. Ein weiterer Hinweis da- 
rauf könnte sein, dass $\mathrm{H}$. Wohlgemuth, aus jüdischer Familie stammend, ihn als Doktorvater gewählt hatte. Auch von E. Stach wissen wir, dass Marlies Teichmüller ihren ehemaligen Lehrer und späteren Chef am Landesamt in Krefeld sehr schätzte (Teichmüller 1989). Lediglich W. Gothan's Verhalten gegenüber Frauen kennen wir etwas genauer. Seine Schülerin, Ingeborg Böwing, berichtete, dass er ein „Kavalier alter Schule" und somit äußerst höflich und zuvorkommend, aber auch herzlich, gewesen sei. Er habe sie auch nach 1945 sehr unterstützt.

\section{Frauen im Bereich Geowissenschaften an der Berliner Universität, erste Phase}

\section{Frauen ohne offizielle Ausbildung}

Während des 19. Jahrhunderts, als Frauen der Zugang zu den Universitäten generell versagt blieb, gab es auch in Berlin naturwissenschaftlich interessierte Frauen, die gewissermaßen im Hintergrund tätig sein mussten (vgl. allgemein hierzu Schiebinger, 1993). Eine dieser frühen, nahezu unbekannten Naturwissenschaftlerinnen war Clara Ehrenberg (1839-1918), Tochter des Universitäts-Professors Christian Gottfried Ehrenberg (1795-1876). Sie half ihrem Vater bei mikropaläontologischen Untersuchungen und zwar mit Beschreibungen und Zeichnungen von Diatomeen und anderen Mikroorganismen, wie Dinoflagellaten und Radiolarien. Später übernahm sie die gesamten kuratorischen Aufgaben an den Sammlungen ihres Vaters, mit dem sie lebte und ihn im Alter betreute (pers. Mitteil. Hannelore Landsberg). Heute befinden sich die Sammlungen am Museum für Naturkunde. Die von Clara angelegten Kataloge bilden noch heute die Grundlage zur Auffindung von Typenmaterial (Lazarus \& Jahn 1998).

Eine weitere bemerkenswerte Frau, die zwar kein Studium in den Geowissenschaften absolviert hatte, aber Außerordentliches für das Geologisch/Paläontologische Institut der Universität geleistet hat, ist Viktorine Helene Natalie von Grumbkow, genannt Ina (1872-1942). Sie begleitete ihren späteren Ehemann, den Vulkanologen und Paläontologen Hans Reck (1886-1937) auf der Suche nach ihrem verschollenen Verlobten, Walther von Knebel, 1908 nach Island (Sapper 1938) und publizierte darüber einen ausführlichen Reisebericht (v. Grumbkow 1909). Maßgeblich war sie auch bei den Ausgrabungen des Instituts am Tendaguru, der bis heute größten, außerordentlich erfolgreichen Grabung nach Dinosaurier, im ehemaligen Deutsch-Ostafrika (Tansania) beteiligt. Ihr Ehemann H. Reck war 1912 nach Ostafrika berufen worden, um die dortigen Grabungen nach Dinosaurier-Skeletten zu leiten. Sie unterstützte ihn tatkräftig und leitete während Reck's Expeditionen die Ausgrabungen ganz allein, bei denen einige hundert Eingeborene als Grabungshelfer beschäftigt waren (v. Grumbkow 1924). 1914 reisten beide wieder nach Ostafrika, um an paläoanthropologischen Grabungen in der OlduveiSchlucht teilzunehmen (Maier 2003), wurden dort aber vom Krieg überrascht, und kehrten nach einigen Monaten in englischer Internierung erst nach dem 1 . Weltkrieg nach Deutschland zurück (v. Grumbkow 1925).

\section{Frauen mit Universitätsabschluss, Deutsches Reich und Weimarer Republik (1906-1933)}

Die erste Frau mit einem geowissenschaftlichen Universitätsabschluss war Helene Wiszwianski (geb. 1881). Sie schloss ihre Dissertation unter Leitung von D. Drygalski (1865-1949, bis 1906 Berlin, später München) und K. W. v. Branca mit dem Thema „Die Faktoren der Wüstenbildung" im Mai 1906 ab und veröffentlichte diese Arbeit im selben Jahr (Wiszwianski 1906). Sie gehörte zu den 22 Promovendinnen, die zwischen 1899 und 1908 jeweils mit Sondergenehmigung an der Philosophischen Fakultät promovierten (Vogt 1998).

Nun vergingen weitere 7 Jahre, ehe Antonie Täuber-Stremme (1882-1961; Abb. 4) die erste Dissertation im Bereich Geologie zu dem Thema „Lage und Beziehungen einiger tertiärer Vulkangebiete Mitteleuropas zu gleichzeitigen Meeren oder großen Seen" bei K. W. v. Branca und A. Penck abschloss und 1913 veröffentlichte. A. Täuber-Stremme, die weitgehend in Berlin aufgewachsen war, hatte zunächst eine Ausbildung als Lehrerin absolviert. Zwischen 1905-1909 unterrichtete sie an öffentlichen und Privatschulen. Danach studierte sie Geologie, Mineralogie und Geographie, daneben auch Volkswirtschaft. 1912 heiratete sie den Privatdozenten am Geologisch/ Paläontologischen Institut, Hermann Stremme, der 1914 einen Ruf als Leiter des MineralogischGeologischen Instituts nach Danzig erhielt und in der Folgezeit die bodenkundliche Kartierung Europas leitete (Stremme 1997). Die Familie wohnte nun in Danzig und hatte 2 Kinder, 1 Tochter und einen Sohn (Herrmann Stremme 


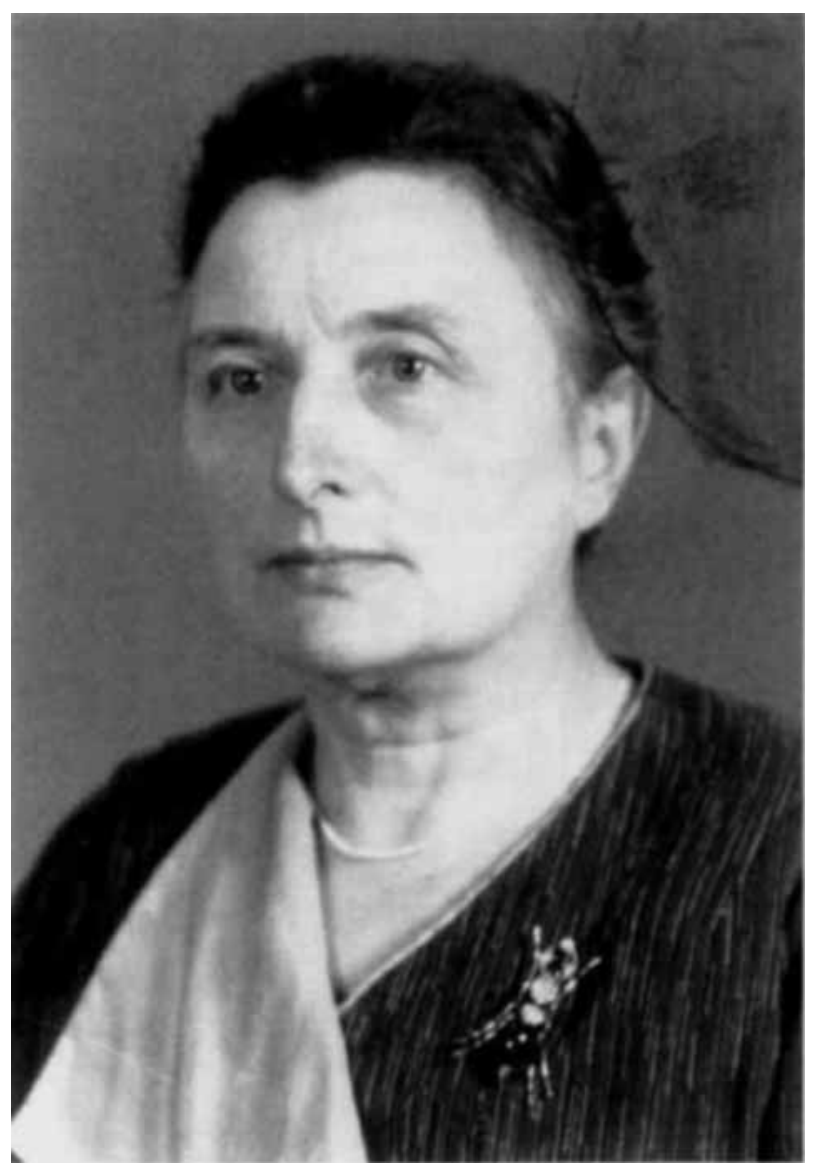

Abb. 4. Antonie Täuber-Stremme, Danzig 1943.

jun., geb. 1916), später Leiter des Geologischen Landesamtes in Kiel. Auch nach ihrer Verheiratung blieb A. Täuber-Stremme aktiv. Sie gründete den Wirtschaftsbund Danziger Hausfrauen, dessen Aufgabe es war, „Frauen für Funktionen im öffentlichen Leben vorzubereiten". Sie war als Abgeordnete in der Danziger Volksvertretung bis 1929 tätig. Während beider Weltkriege hat sie vertretungsweise die Geschäfte ihres Mannes am Mineralogisch-Geologischen Institut in Danzig geleitet. Nach 1945 erstellte sie als Assistentin am Geographischen Institut der Humboldt-Universität Wirtschaftskarten für den Berliner Magistrat.

Bereits 1914 wurde eine weitere Dissertation mit einem petrologisch-geologisch-mineralogischen Thema von Dorothea Mesch (geb. 1890) vorgelegt: „Die Basalte des Kamerungebirges und des Gebietes zwischen Kamerungebirge und Elefantensee". Die Gutachter waren T. Liebisch und K. W. v. Branca.

Dann aber folgte eine längere Pause. Zwischen 1914 und 1925 promovierte an der Berliner Universität keine Frau in den Geowissenschaften. Dies ist um so erstaunlicher, als während des ersten Weltkrieges die Zahl der Studentinnen und die Zahl der Promovendinnen erheblich zunahm, da die Frauen als "akademische Reservearmee" die Lücken der in den Krieg eingezogenen Männer schließen durften. Die Situation in den Geowissenschaften hing möglicherweise mit den Ordinariatswechseln in den betreffenden Fächern zusammen. Solche Veränderungen sind allgemein für Studienabschlüsse ungünstig.

Erst 1925 folgte wieder eine Promotion, und Charlotte Möller (Lotte Möller) (1893-1973; Abb. 5) wurde eine der seltenen Ausnahmen. Sie promovierte mit der Arbeit "Die Deviation bei Strommessungen im Meere" bei A. Merz und A. Penck, die ihre Leistung mit dem selten vergebenen „valde laudabile" bewerteten. Auch die Prüfung bestand sie mit "magna cum laude“. Lotte Möller blieb ihrer Universität 20 Jahre verbunden, bis 1945: als Promovendin, Assistentin, Privatdozentin (1929), Kustodin, außerordentliche Professorin (1935) bzw. außerplanmäßige Professorin (1939-1945). Als eine der wenigen Wissenschaftlerinnen wurde sie 1940 Mitglied der angesehenen und ältesten Akademie Deutschlands, der Leopoldina (Vogt 1996, S. 52-54; Brosin 1999).

Lotte Möller hatte bereits 1929, nur 4 Jahre nach Abschluss der Promotion, im Fach Hydrologie habilitiert und war eine der 12 Privatdozentinnen an der Philosophischen Fakultät der Berliner Universität (Vogt 1999). Mit ihrer Habilitationsschrift war sie an der Leitung und Aus-

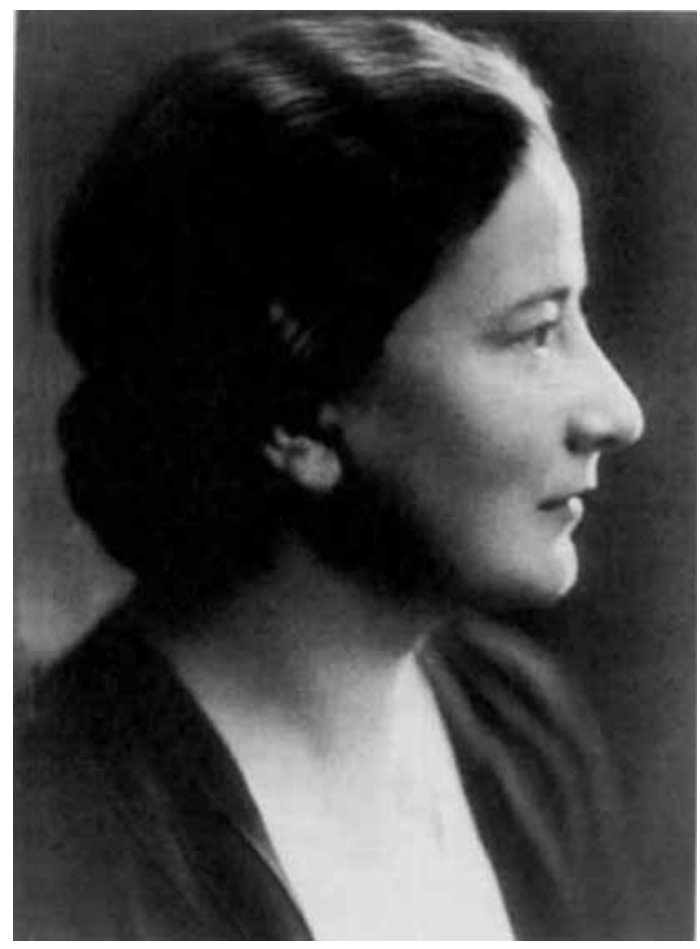

Abb. 5. Lotte Möller 1932 (aus: Brosin 1999). 
wertung der Deutschen Atlantischen Expedition im Indischen Ozean mit dem Vermessungs-Schiff „Meteor“ beteiligt, durfte aber als Frau das Schiff nicht betreten. Sie war von A. Merz, A. Penck und später von N. Krebs unterstützt worden, was ihrer Laufbahn an der Philosophischen Fakultät sehr förderlich war. Sie selbst betreute ebenfalls einige Promotionen, darunter auch solche von Frauen. In der Nazi-Zeit „kooperierte“ sie mit NS-Stellen, u. a. mit der Organisation Todt und der Wehrmacht. Nach dem Überfall auf die Sowjetunion plante sie, in den besetzten Gebieten hydrologische Untersuchungen durchzuführen. Dies misslang auf Grund einer Erkrankung (Sumpffieber) und führte später zu ihrer Entlassung von der Berliner Universität 1945 gemäß den alliierten Bestimmungen zur Entnazifizierung. 1950 erhielt sie ein Angebot, die Leitung der Abteilung Meereskunde des seehydrographischen Dienstes der DDR zu übernehmen. Aber sie zog es vor, ab 1952 einen unbezahlten Lehrauftrag zu übernehmen. 1956 habilitierte sie sich an der Universität Göttingen um.

1928 promovierte Hertha Sieverts-Doreck (1899-1991; Abb. 6). Sie wurde in Völklingen bei Saarbrücken als Tochter eines Chemikers und älteste von drei Schwestern geboren. Nach Schulabschluss 1916 in Berlin folgte eine pädagogische Ausbildung. 1921 erhielt sie die Lehrbefähigung für Lyceen und Mittelschulen in Preußen (Haude 1992). Danach studierte sie Mathematik und Naturwissenschaften in Berlin und Innsbruck. Sie promovierte 1928 an der Berliner Universität mit der Arbeit „Über die Crinoidengattung Marsupites" bei J. F. Pompeckj und K. Zimmer, dem Leiter des Zoologischen Instituts

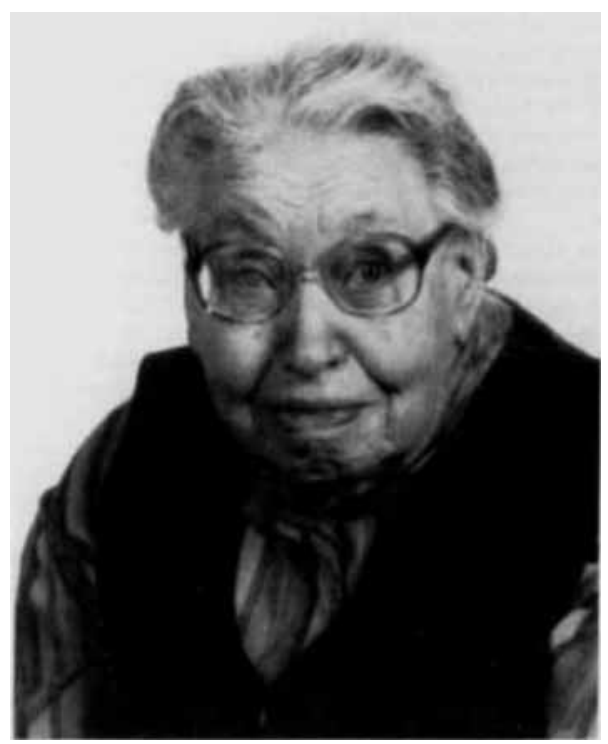

Abb. 6. Hertha Sieverts-Doreck 1991 (aus: Haude 1992). am Museum für Naturkunde. Danach erhielt sie nur kurzzeitige Anstellungen an der TH Berlin und an der TH Karlsruhe. Ab 1930 war sie Privatassistentin von Johannes Wanner, der zu dieser Zeit am Institut für Angewandte Geologie in Bonn arbeitete. 1936 schloss sie eine Ausbildung zur Landesgeologin an der Preußischen Geologischen Landesanstalt in Berlin mit Geländearbeiten $\mathrm{ab}$ und heiratete W. Doreck. Sie zogen nach Stuttgart, wo zwei Töchter geboren wurden. Hertha Sieverts-Doreck veröffentlichte etwa 70 Publikationen zum Thema Crinoiden. Sie wurde auch als Spezialistin für die Standardwerke des "Treatise on Invertebrate Paleontology" (Moore \& Teichert (eds) 1978) herangezogen, eine ausgesprochen ehrenvolle Aufgabe.

Ebenfalls 1928 promovierte Gertrud DennerBobek (1898-2000; Abb. 7). Nach einem Studium der Geographie und Geologie beendete sie ihre Dissertation „Über die Ödländereien Deutschlands" und arbeitete als Sekretärin bei der Gesellschaft für Erdkunde. Sie hatte 1926 den Physikochemiker Dr. Felix Bobek (18981938) geheiratet und bekam durch ihn engen Kontakt zur Kommunistischen Partei Deutschlands (KPD). Seit 1933 leisteten sie Widerstand gegen das NS-Regime. F. Bobek wurde verhaftet. 1937 zum Tode verurteilt und 1938 hingerichtet. G. Bobek konnte mit ihren beiden Mädchen in die Sowjetunion flüchten, wo sie in Moskau zunächst in einem Internationalen Agrarinstitut (1936-1940), später am Geographischen Institut der Sowjetischen Akademie (1940-1944) arbeitete. $1945 \mathrm{kam}$ sie mit politischem Auftrag in die Sowjetisch besetzte Zone (SBZ) bzw. in die Deutsche Demokratische Republik (DDR) zurück, und war ausschließlich in politischen Funktionen tätig (vgl. Bobek 1998).

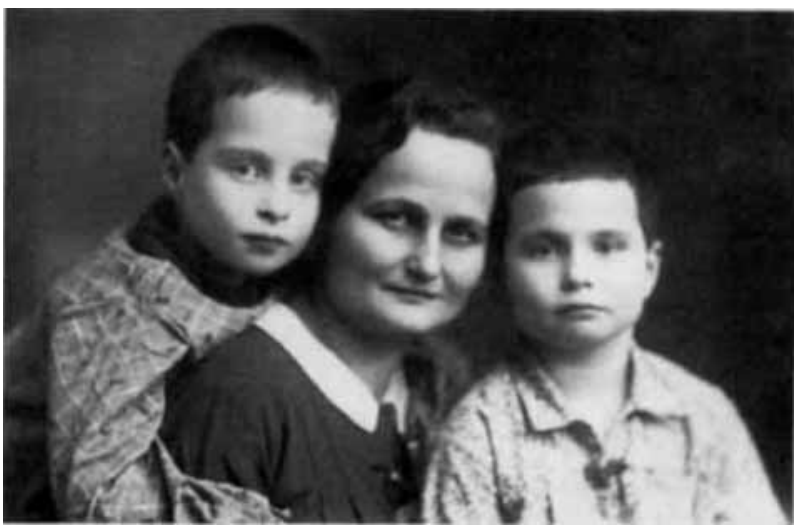

Abb. 7. Gertrud Bobek mit ihren beiden Töchtern 1937 in Moskau (aus: Erinnerungen an mein Leben 1998). 


\section{Die Zeit des Nationalsozialismus (1933-1945)}

Die Veränderungen, $d . h$. Verschlechterungen für Frauenstudium und Frauenberufstätigkeit im akademischen und außerakademischen Bereich waren auf Grund der NS-Bedingungen erheblich (Bronnen 1998). Allgemein ging die Zahl der Studentinnen und Promovendinnen zurück, Frauen mit jüdischem Familienhintergrund waren sofort Diskriminierungen ausgesetzt (Crane 2003). Mit dem so genannten „Gesetz zur Wiederherstellung des Berufsbeamtentums" vom 7. April 1933 wurden politische Gegner(innen), sowie nach der NS-Definition "nicht-arische" Mitglieder der Universität entlassen (Vogt 2001). So blieb von den sechs Naturwissenschaftlerinnen an der Berliner Universität, die als Assistentinnen angestellt waren, eine übrig. Sechs der 10 Privatdozentinnen verloren ihre Stelle. Zwischen 1933 und 1938/39 wurden kaum Assistentinnen eingestellt, die Habilitation von Frauen war unerwünscht. Mit Beginn des zweiten Weltkrieges änderte sich die Politik erneut. Wie im ersten Weltkrieg, und im Gegensatz zur offiziellen NS-Propaganda, waren nun Frauen wieder erwünscht, als Assistentinnen willkommen, sogar als Privatdozentinnen für die Berliner Universität (vgl. Vogt 1999). Deshalb promovierten vergleichsweise viele Frauen zwischen 1936 und 1945 an der Mathematisch-Naturwissenschaftlichen Fakultät, die 1936 durch Teilung aus der Philosophischen Fakultät entstanden war.

Ferdinande Michael war eine Studentin von Walther Gothan. Möglicherweise studierte sie Geologie an der Technischen Hochschule Berlin, da über sie keine Unterlagen im Archiv der Friedrich-Wilhelms-Universität aufzufinden sind. Ferdinande Michael veröffentlichte 1936 ihre Dissertation über unterkretazische pflanzliche Makrofossilien im Jahrbuch der Preussischen Geologischen Landesanstalt, die noch Jahrzehnte später zitiert wurde.

Nur zweimal, 1934 und 1943, wurde eine Promotion im Fach Geophysik abgeschlossen. Aber beide Fälle unterschieden sich wesentlich, sowohl bezüglich Thema und Betreuung, als auch bezüglich der späteren Berufstätigkeit.

Gertrud Kobe (1905-1995) schloss 1934 bei Albert Defant und Heinrich von Ficker die Arbeit „Der hydrographische Aufbau und die dadurch bedingten Strömungen im Skagerrak“ ab. G. Kobe war von 1934 bis 1938 Assistentin an der Universität, zuerst $1 \mathrm{Jahr}$ am Institut für Meereskunde - an dem auch L. Möller tätig war -, danach am Meteorologischen Institut der
Universität unter $H$. von Ficker. Da sie die Nazionalsozialisten ablehnte, empfahl ihr $\mathrm{H}$. von Ficker den Weggang von der Universität, zumal auch er sein Institut aufgab und ein Observatorium der Kaiser-Wilhelm-Gesellschaft übernahm. Sie ging - und das mag überraschend klingen zum Militär, von 1938 bis 1945 war sie Referentin, später Gruppenleiterin, am Marineobservatorium in Wilhelmshaven. Sie mußte ihr Forschungsgebiet von der Geophysik zur Meteorologie wechseln. Da sie keine NS-Anhängerin war, wurde sie nach Wiedereröffnung der Berliner Universität 1946 sofort eingestellt und blieb bis zu ihrer Emeritierung 1965 hier tätig (Vogt 2000c: 136-141).

Ganz anders verlief die Karriere von Erna Hein (geb. 1902). Sie schloss 1943 ihre Arbeit „Hydrographische Untersuchung des Heinitzsees in Rüdersdorf/Mark“ $a b$, die von L. Möller und N. Krebs begutachtet wurde. Sie promovierte als 41-jährige Lehrerin und blieb mit großer Wahrscheinlichkeit auch danach im Schuldienst.

Hilde Wohlgemuth, geb. Heimann, schloss ihre Promotion 1935 in den Fächern Geographie/ Geologie und Mineralogie ab. Sie stammte aus einer jüdischen Wuppertaler Kaufmanns-Familie, rettete sich in der NS-Zeit nach Schweden und heiratete (heute Rohlen-Wohlgemuth). Sie hat sich wahrscheinlich gut in Schweden eingelebt, denn sie veröffentlichte Aufsätze, Bücher und Bibliographien zur jüdischen Geschichte, sowohl in schwedischer, als auch in deutscher Sprache (u. a. Rohlen-Wohlgemuth 1994).

1941 schloss Marie-Luise (Marlies) Teichmüller (1914-2000; Abb. 8), die erfolgreichste aller deutschen Geologinnen vor den 1970er Jahren, ihre Dissertation „Der Feinbau amerikanischer Kohlen im Anschliff und Dünnschliff. Ein Vergleich kohlenpetrographischer Untersuchungsmethoden" ab, die im "Jahrbuch der Reichsstelle für Bodenforschung " 1941 erschien. Die Arbeit begutachteten die Geologen Erich Stach und Hans Stille äußerst positiv. Die Publikation in einem angesehenen Journal bewies die Qualität. Marlies Teichmüller war seit 1938 mit dem habilitierten Geologen Rolf Teichmüller verheiratet. Nur weil der Krieg beachtliche Lücken schuf, durfte sie als verheiratete Frau tätig sein - entgegen der noch immer offiziell propagierten Politik von „Küche-Kinder“. Auch später blieb M. Teichmüller berufstätig, von 1947 bis 1979 am Geologischen Landesamt Nordrhein-Westfalen in Krefeld, wo sie in späteren Jahren eine Stelle als Geologiedirektorin inne hatte. In ihrem Hauptarbeitsgebiet, Kohlenpetro- 


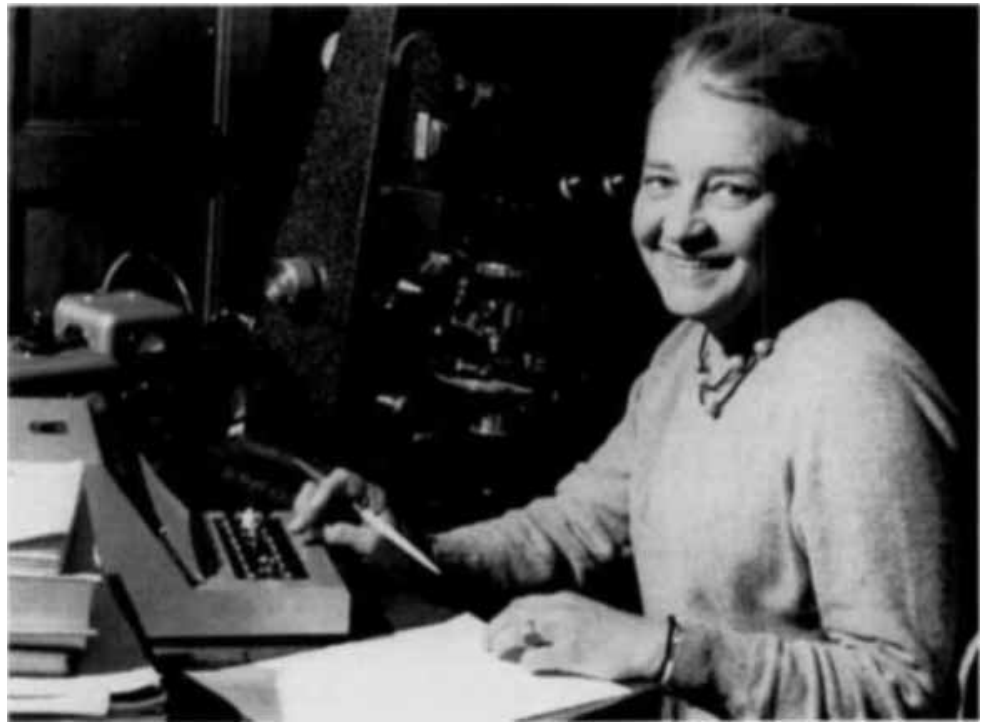

Abb. 8. Marlies Teichmüller, in ihrem Büro in Krefeld (60er Jahre?).

logie, veröffentlichte sie 190 Arbeiten. Sie war Co-Autorin bei drei Handbüchern zur Kohlenpetrologie, darunter noch jüngst bei dem Buch „Organische Petrologie“ (Taylor et al. 1998). Sie erhielt mehrere internationale Preise für ihr Werk. Eine detaillierte Studie zum Leben und Werk von M. Teichmüller ist geplant (Mohr \& Vogt in prep.)

Eine weitere Arbeit, die im Reichsamt für Bodenforschung angefertigt wurde, war die Dissertation von Ingeborg Böwing (geb. 1911; Abb. 9). Noch im März 1945 beendete sie ihre Arbeit „Das Alter der samländischen und ostpommerschen Braunkohlenformation auf Grund der Flora" bei W. Gothan und Ludwig Diels (1874-1945), dem Direktor des Botanischen

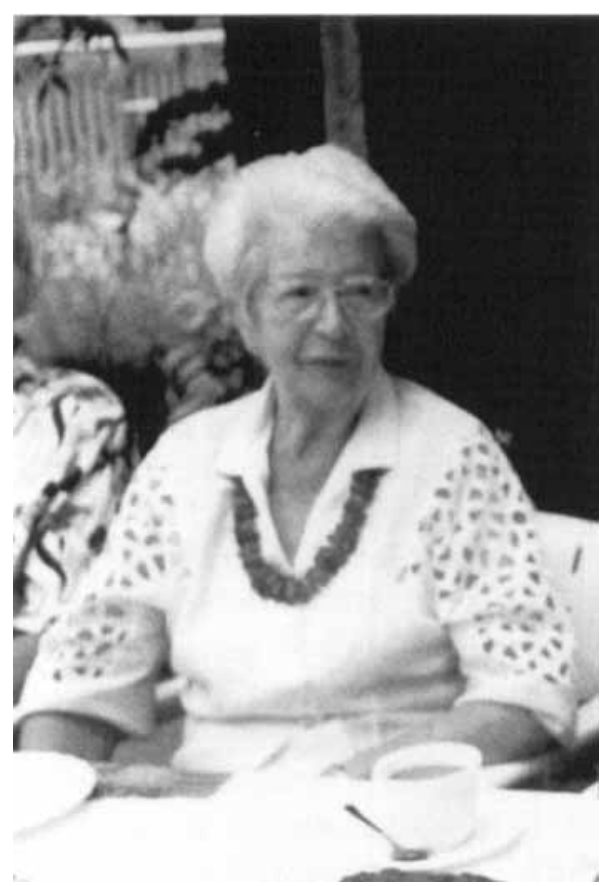

Abb. 9. Ingeborg Böwing, Kleinmachnow 2001.
Instituts und Gartens in Dahlem. Auch sie war verheiratet und Mutter von 4 Kindern. Aber obwohl sie die wissenschaftliche Arbeit begeisterte, mußte sie 1945 ihre Pläne, weiter in diesem Fachbereich tätig zu sein, aufgeben. Nach dem plötzlichen Tod ihres Mannes hatte sie die Leitung seiner Apotheke zu übernehmen, die Familie zu ernähren, den Kindern ein Studium zu ermöglichen. Ihre wissenschaftlichen Ambitionen hatten demgegenüber zurück zu treten (Interview, 12. 11. 1998).

\section{Analyse}

Die Primärdaten wurden in Tabellen zusammengefasst. Tabelle 1 listet die Anzahl aller Promovendinnen in den Naturwissenschaften an der FWU Berlin auf. In einem gesonderten Anhang (Appendix) werden alle uns zur Verfügung stehenden Daten in Englisch beigegeben.

\section{Studienfächer}

Vor 1945 wählten die meisten Frauen im Bereich Geowissenschaften die Geographie als Hauptfach, insgesamt 21mal. Davon entschieden sich 9 Frauen für physische Geographie/Geologie. 3 Frauen wählten Geologie/Paläontologie, 1 Mineralogie, 2 Paläobotanik und 2 Geophysik, also insgesamt 17 Frauen, deren Daten im folgenden ausgewertet werden.

\section{Karriere und Familien-Status}

Fast alle Frauen, die vor 1945 studiert hatten, kamen aus bürgerlichen, mitunter aus akademischen Familien. Die Berufe der Väter waren u. a. 
Pharmazeut, Ingenieur, Staatsbeamter, Kaufmann, Lehrer. Lediglich eine der Studentinnen stammte aus einer Handwerkerfamilie.

Von 10 der 17 bis 1945 promovierten Frauen ließen sich die Spuren weiter verfolgen. Drei Frauen, L. Möller, G. Kobe und M. Teichmüller gestalteten ihre Karriere durchgängig in ihrem Studienfach. In einigen dieser Fälle kam es zu Unterbrechungen, bedingt durch die NS-Zeit (Frau Hilde Wohlgemuth) oder durch familiäre Verpflichtungen.

Von den uns bekannten Promovendinnen war eine Lehrerin (E. Hein) und hat mutmaßlich diesen Beruf auch weiterhin ausgeübt, zwei gaben wegen ihrer Heirat den Beruf auf, eine unterstützte in der Folgezeit ihren Mann (A. TäuberStremme) und die zweite blieb weiterhin privat als Wissenschaftlerin tätig (Sieverts-Doreck). Zwei Promovendinnen hatten nach dem Tod ihres Mannes die Familie zu ernähren und mussten deshalb das Arbeitsgebiet wechseln (G. Bobek und I. Böwing).

$\mathrm{Ob}$ die Promovendinnen später heirateten, ist oft nicht zu beantworten, wenn sich die Spuren nach dem Universitäts-Abschluss verlieren. Einige Frauen heirateten nach Abschluss des Studiums, andere waren schon vor Abschluss der Promotion (vgl. M. Teichmüller oder I. Böwing) verheiratet. Nur wenige der Promovendinnen hatten zum Zeitpunkt des Studiums bzw. der Promotion bereits Kinder. Zwei Promovendinnen hatten Kinder und arbeiteten trotzdem nach dem Tod ihrer Ehemänner weiter, nämlich $G$. Bobek und I. Böwing. Dies scheint die Annahme zu bestätigen, dass Karriere und Familie nicht diametral verschieden sein müssen oder sich ausschließen. Denn unter „unseren“ erfolgreichsten Geowissenschaftlerinnen waren sowohl ledige, als auch verheiratete. Allerdings handelte es sich in den Fällen verheirateter und erfolgreicher Geowissenschaftlerinnen oft um jene, deren Ehepartner ebenfalls auf diesem Gebiet tätig waren (M. Teichmüller). Die Unterstützung erfolgte also eindeutig durch den Kollegen, der zugleich Partner war (s. Forscher-Ehepaare, Pycior et al. 1996).

Insgesamt kann konstatiert werden, dass ledigleich drei der Frauen eine ungebrochene Karriere in ihrem Beruf verfolgten, zählt man die Lehrerin E. Hein hinzu, waren es vier.

\section{Zur Wirkungsgeschichte}

Zum gegenwärtigen Zeitpunkt ist es schwierig, die Auswirkung der Arbeiten „unserer“ Geowis- senschaftlerinnen einzuschätzen bzw. zu bewerten. Dafür sind mehrere Gründe verantwortlich. Erstens gab es Geowissenschaftlerinnen nennenswert erst $a b$ den späten 20er Jahren, d. h. nicht einmal seit 80 Jahren. In dieser Zeit können die wenigen Geowissenschaftlerinnen nicht ernsthaft mit der deutlich höheren Zahl der männlichen Kollegen verglichen werden, jede bleibt ein Einzelfall, bestenfalls eine Ausnahme. Zweitens ist auf Grund der schlechteren Karrierebedingungen für Wissenschaftlerinnen für den hier untersuchten Zeitraum, ein Vergleich wiederum nur bedingt oder zu ungunsten der Frauen möglich. An den schlechteren Berufschancen lag es z. B., dass oft nur die Dissertation der betreffenden Frauen als die einzige zu bewertende wissenschaftliche Leistung nachweisbar ist. Dies ist in der Regel die erste selbständige wissenschaftliche Arbeit. Oft wurde diese nicht publiziert (was für viele Dissertationen vor 1945 gilt), ist also nur noch in Archiven zugänglich und kann deshalb nicht von der aktuellen Forschung rezipiert werden. Damit ist die Wirkung aber höchst eingeschränkt.

Jedoch gibt es einige Wissenschaftlerinnen, denen eine überaus erfolgreiche Karriere gelang. An erster Stelle ist L. Möller zu nennen, die aktiv an der Universität in der Lehre tätig war und außerdem zwei Promovendinnen betreute (Tab. 2). Auch G. Kobe forschte und lehrte nach 1945 an der Berliner Universität. M. Teichmüller war nicht nur Direktorin, sondern beeinflusst bis heute mit der großen Zahl ihrer Publikationen ihr Arbeitsgebiet. Ein anderes Beispiel ist die Wissenschaftlerin H. Sieverts-Doreck, die zwar seit ihrer Heirat 1936 nie offiziell angestellt, aber dennoch wissenschaftlich äußerst produktiv war. Aufgrund ihrer vielen Publikationen zur Biologie der Crinoiden und als Co-Autorin wichtiger Handbücher wird sie sicher auch in Zukunft zitiert werden.

Die drei Beispiele zeigen, dass es einzelnen Wissenschaftlerinnen - den Ausnahmen - gelang, erfolgreich und anerkannt wissenschaftlich tätig zu sein, sowohl im akademischen Bereich (L. Möller an der Universität), als auch im außerakademischen Bereich (M. Teichmüller an der Landesanstalt) und sogar ohne reguläre Anstellung oder institutionelle Einbindung $(\mathrm{H}$. Sieverts-Doreck). Wesentlich für die Wirksamkeit der Wissenschaftlerinnen waren (und sind) ihre Publikationen, insbesondere das Verfassen von Monographien. 


\section{Ergebnisse}

Basierend auf unserem beschränkten Datensatz und der Analyse, können wir folgende Feststellungen treffen. Frauen begannen in Berlin, ähnlich wie im übrigen Deutschland, in Westeuropa, U.S.A. und Australien, aber im Gegensatz zu Russland, spät mit einem geowissenschaftlichen Studium. Neben dem späten Einsetzen ist die zweite Irregularität gegenüber anderen Studienfächern die geringe Anzahl der Geowissenschaftlerinnen. Dennoch waren vor 1933, bedingt durch die zentrale Funktion der Universität in der Reichshauptstadt, die Möglichkeiten für Frauen generell relativ gut. Allerdings entsprach die Zahl der tatsächlich ausgebildeten Frauen, im Bereich Geowissenschaften bei weitem nicht den Kapazitäten. Dies hatte seine Gründe in der konservativen Struktur der preußischen Institutionen. Geographie wurde am häufigsten als Studienfach gewählt, weil dort mindestens die Möglichkeit bestand, im Schuldienst eine Stelle zu finden, wenngleich auch Geologie an einigen Gymnasien gelehrt wurde. Geologie wurde nur dreimal im Hauptfach studiert. Hier wird die geringe Zahl der weiblichen Studierenden durch die enge Verbindung von Geologie und Bergbau, einem absolut frauenfeindlichen Bereich, bedingt gewesen sein. Nochmals erschwert wurde die Situation während der Weltwirtschaftskrise ( $a b$ 1929) und in den ersten Jahren des NS-Regimes. Erst mit der Vorbereitung und während des 2. Weltkrieges wuchsen die Berufschancen, als Frauen die Plätze der Männer einnehmen "durften".

Heirat und Kinder erwiesen sich als nicht hinderlich für die weitere Entwicklung der Berufskarriere. In den Fällen, in denen der Ehepartner auch Kollege war, wirkte sich dies eher fördernd auf die Karriere aus (A. Täuber-Stremme, M. Teichmüller).

\section{Danksagung}

Wir möchten all jenen unseren Dank aussprechen, die uns bei der Abfassung dieses Aufsatzes geholfen haben: R. Daber, M. Guntau, H. Kahlert, P. Krüger, L. Rüffle. St. Schultka (alle Berlin). H. Landsberg und S. Hackethal von der Historischen Abteilung des MfN Berlin überließen uns dankeswerterweise Bildmaterial. Dem Ehepaar U. und D. Todtenhaupt (Berlin) danke ich für die Information über I. v. Grumbkow und die Überlassung mehrerer Bücher, die für die vorliegende Arbeit genutzt wurden. M. Teichmüller und deren Schwester, Frau I. Hirdes, schrieben ausführliche Briefe zum (wissenschaftlichen) Werdegang von M. Teichmüller. H. Stremme (Kiel) lieferte uns mündlich und schriftlich viele Hintergrundinformationen zum Lebenslauf seiner Mutter.
I. Böwing (Kleinmachnow) war so freundlich, uns mündlich Auskunft uber Ihren Lebensweg zu geben. Zwei Gutachterinnen sei herzlich für ihre Mühe und teilweise ausführlichen Kommentare gedankt, die wesentlich zur Verbesserung des Manuskripts beigetragen haben.

\section{Literatur}

Asen, J. 1955. Gesamtverzeichnis des Lehrkörpers der Universität Berlin. 1810-1945. 279 pp., Otto Harrassowitz, Leipzig.

Bobek. G. 1974. Schwerer Anfang in der befreiten Heimat. Im Zeichen des roten Sterns. Erinnerungen. pp. 427-440, Dietz-Verlag, Berlin.

- 1998. Erinnerungen an mein Leben. 320 pp., Tauchaer Verlag, Taucha.

Boedeker, E. \& Meyer-Plath, M. 1974. 50 Jahre Habilitation von Frauen in Deutschland. Eine Dokumentation von 1920-1970. 373 pp., Verlag Otto Schwartz \& Co., Göttingen.

Bronnen. B. (ed.): Geschichten vom Überleben. Frauentagebücher aus der NS-Zeit. 247 pp., Verlag Beck, München.

Brosin. H.-J. 1999. Lotte Möller (1893-1973) und die gewässerkundlichen Arbeiten am Institut für Meereskunde Berlin. - Historisch-Meereskundliches Jahrbuch, Deutsches Meeresmuseum Stralsund 6: 19-34.

Crane, C. 2000. Divided lives: Jewish-christian women in Nazi Germany. 352 pp., Palgrave Macmillan, New York.

Grumbkow, I. v. 1909. Isafold. Reisebilder aus Island. 202 pp., Dietrich Reimer, Berlin.

- 1924. Mit der Tendaguru-Expedition im Süden von Deutsch-Ostafrika. 73 pp., Dietrich Reimer, Berlin.

- 1925. Auf einsamen Märschen im Norden von DeutschOstafrika. 125 pp., Dietrich Reimer, Berlin.

Hammerstein, N. 1989. Die Johann Wolfgang Goethe-Universität Frankfurt am Main. Von der Stiftungsuniversität zur staatlichen Hochschule. Vol. 1: 1914 bis 1950. 917 pp., Alfred Metzler Verlag, Neuwied/Frankfurt/M.

Haude, R. 1992. Hertha Doreck. Nachruf. - Paläontologische Zeitschrift 66: 1-7.

Heck, H. L. 1966. Friedrich Solger zum Gedenken. - Berichte der deutschen Gesellschaft für geologische Wissenschaften 11 (6): 745-746.

Hennig. E. 1930. Josef Felix Pompeckj. - Centralblatt für Mineralogie, Abteilung B 9: 353-366.

Hoyermann, Th. 1917. Über Dorsenetia Buckman und Ammonites Romani Opp. (unter besonderer Berücksichtigung des Vorkommens bei Geerzen im Hilsgebiet). Unveröffentlichte Dissertation, 64 pp., Eberhard-KarlsUniversität Tübingen.

Kasig, W. 1983. Marlies und Rolf Teichmüller. - Nachrichten der deutschen geologischen Gesellschaft 29: 55-66.

Katalog Museum für Verkehr und Technik 1996. Aufgetaucht. Das Institut und Museum für Meereskunde im Museum für Verkehr und Technik Berlin. - Schriftenreihe des Museums für Verkehr und Technik 15: $171 \mathrm{pp}$., Nicolai'sche Verlagsbuchhandlung, Berlin.

Kölbl-Ebert, M. 1997. Charlotte Murchinson née Hugonin 1788-1869. - Earth Sciences History 16 (2): 39-43.

- 2001. On the origin of women geologists by means of social selection: German and British comparison. - Episodes 24: 182-193.

Kohring, R. 1997. Senckenbergische Forscher: Tilly Edinger (1897-1967). - Natur und Museum 127: 391-410.

Lazarus, D. \& Jahn, R. 1998. Using the Ehrenberg collection. - Diatom Research 13 (2): 273-291.

Lehnert. E. 1999. Ausschluß-Aufbruch-Zulassung. Von der geduldeten Gasthörerin zur Studentin. In Meyer, $\mathrm{H}$ (ed.). Frauen an der Humboldt-Universität. 1908-1998. Humboldt Universität, Heft 99: 7-19. 
Lenz, M. 1910. Geschichte der Königlichen Friedrich-Wilhelms-Universität zu Berlin. 3. Bd., 536 pp., Verlag der Buchhandlung des Waisenhauses, Halle a. d. Saale.

Maier, G. 2003. African dinosaurs unearthed. The Tendaguru expeditions. $280 \mathrm{pp}$., Indiana University press, Bloomington.

Michael, F. 1936. Paläobotanische und kohlenpetrographische Studien in der nordwestdeutschen Wealdenformation. Abhandlungen der Preußischen Geologischen Landesanstalt, Neue Folge 166: 1-79.

Mohr, B. A. R. \& Schultka, St. 1998. Goethe, Gothan, Gimm und andere. Über die paläobotanischen Sammlungen und Sammler des Museums für Naturkunde in Berlin. Museumsjournal 12 (1): 61-63.

Mohr, B. A. R. \& Vogt, A. (2001): German woman paleobotanists from the 1920 s to the early 1970 s - or why did this story start so late? - Earth Sciences History 20: $13-43$

Moore, R. C. \& Teichert, C. (eds) 1978. Treatise on Invertebrate Paleontology. Echinodermata 2 (1). 401 pp., Geological Society of America, University of Kansas, Lawrence.

Nalivkin, Dmitrij Vasil'evič 1979. Naši pervye ženščiny-geologi. (Unsere ersten Geologinnen). 215 pp., Leningrad, Nauka.

Pycior, H. M., Slack, N. G. \& Abir-Am, P. G. 1996. Creative Couples in the Sciences. $369 \mathrm{pp}$,, Rutgers University Press, New Brunswick, New Jersey.

Reck, R. \& Kaufer, M. 1997. Frauenbewegungen in Russland. - Wissenschaftsladen Innsbruck Heft 31/32: $28 \mathrm{pp}$.

Reinsch, H. \& Lehnert, E. 1996. Zu den noch nicht gehobenen Schätzen des Berliner Universitätsarchivs - dargestellt am Beispiel der Anfänge des Frauenstudiums. In Zur Geschichte des Frauenstudiums und weiblicher Berufskarrieren an der Berliner Universität 1918-1933. Zentrum für interdisziplinäre Frauenforschung ( $\mathrm{ZiF})$ : $18-33$.

Rohlen-Wohlgemuth, H. 1994: Die Familie Alexander Levy in Eschwege. - Eschweger Geschichtsblätter, Heft 5: $103 \mathrm{pp}$.

Rothe, P. 1997. Ilse Plewe (geb. Voelcker). - Jahresberichte und Mitteilungen des oberrheinischen geologischen Vereins, Neue Folge 79: 39-43.

Sapper, K. 1938. Hans Reck. - Zeitschrift für Vulkanologie 17 (4): $225-232$.

Schwarzer, T. F. \& Crawford, M. L. 1977. American women in geology. A historical perspective. - Geology 5: 493-504.

Sieverts, H. 1927: Über die Crinoidengattung Marsupites. Abhandlungen der preußischen geologischen Landesanstalt, Neue Folge 108: 1-73.

Soden, E. von 1913. Das Frauenbuch. Frauenberufe und -Ausbildungsstätten. 238 pp., Franck'sche Verlagshandlung, Stuttgart.

Stach, E. 1935. Lehrbuch für Kohlenpetrographie. 293 pp., Bornträger, Berlin.

Stober, V. \& Riedmaier, T. 1987. Frauen in den Naturwissenschaften. - Begleitheft zur Ausstellung an der Universität Karlsruhe: $51 \mathrm{pp}$.

Stremme, H. E. 1997. Preparation of the collaborative soil maps of Europe, 1927-1937. In D. H. Yaalon \& S. Bercowicz (eds). History of Soil Sciences - International Perspectives. Advances in Geoecology 29: 145-158, Catena Verlag, Reiskirchen

Suiter, M. J. 1992. Women in Geoscience: A resource to develop. - Geotimes 37 (1): 14-17.

Täuber, A. 1914. Lage und Beziehung einiger tertiärer Vulkangebiete Mitteleuropas zu gleichzeitigen Meeren oder großen Seen. - Neues Jahrbuch für Mineralogic Geologie und Paläontologie, Beiheft 36: 413-490.

Taylor, G. H., Teichmüller, M., Davis, A. Diessel, C. F. K., Littke R. \& Robert, P. 1998. Organic Petrology. 704 pp., Gebrüder Bornträger, Berlin, Stuttgart.
Teichert, C. 1986. An early German supporter of Continental drift. - Earth Sciences History 5 (1): 134-136.

Teichmüller, M. 1941. Der Feinbau amerikanischer Kohlen im Anschliff und Dünnschliff. Ein Vergleich kohlepetrographischer Untersuchungsmethoden. - Jahrbuch der Reichsanstalt für Bodenforschung 61: 20-55.

- 1989. Erich Stach (1896-1987), a life devoted to coal petrology. - International Journal of Coal Geology 14: $1-4$.

Turner, S. 1994. Women in Palaeontology in Australia. In Branagan D. F. \& G.H. McNally (eds). Useful and curious Geological enquiries beyond the world. Pacific-Asia historical Themes. $19^{\text {th }}$ International Symposium of the International Commission for the History of Geological Sciences: $248-250$, Sydney.

Villinger, E. 2001. Frauen im Oberrheinischen Geologischen Verein. - Jahresberichte und Mitteilungen des Oberrheinischen Geologischen Vereins, Neue Folge 83: 379424.

Vogt, A. 1996. Zu den naturwissenschaftlichen Promotionen von Frauen an der Philosophischen Fakultät der Berliner Universität zwischen 1898 und 1945 - Überblick und Einzelbeispiele. In Zur Geschichte des Frauenstudiums und weiblicher Berufskarrieren an der Berliner Universität. ZiF: 34-57.

- 1997. Findbuch (Index Book). Die Promotionen von Frauen an der Philosophischen Fakultät von 1898 bis 1936 und an der Mathematisch-Naturwissenschaftlichen Fakultät von 1936 bis 1945 der Friedrich-Wilhelms-Universität zu Berlin sowie die Habilitationen von Frauen an beiden Fakultäten von 1919 bis 1945. - Max-Planck-Institut für Wissenschaftsgeschichte, Heft 57: $120 \mathrm{pp}$.

- 1998. Die Spielregeln der Objektivität. Die ersten Promotionen und Promotionsversuche von Frauen an der philosophischen Fakultät der Berliner Friedrich-Wilhelms-Universität 1898-1908. In Bleker, J. (ed.). Der Eintritt der Frauen in die Gelehrtenrepublik. Zur Geschlechterfrage im akademischen Selbstverständnis und in der wissenschaftlichen Praxis am Anfang des 20. Jh. - Abhandlungen zur Geschichte der Medizin und Naturwissenschaften 84: $31-48$.

- 1999. Aufbruch und Verdrängung. Wissenschaftlerinnen an der Berliner Universität zwischen 1918 und 1945/46. In Meyer. H. (ed.). Frauen an der Humboldt-Universität. 1908-1998. - Humboldt-Universität zu Berlin, Heft 99: 21-48.

- 2000a. Die ersten Karriereschritte - Physikerinnen im Berliner Raum zwischen 1900 und 1945. In Dieckmann, E., Schöck-Quinteros, E. \& Dauks, S. (eds). Barrieren und Karrieren. Die Anfänge des Frauenstudiums in Deutschland. pp. 195-230, trafo Verlag, Berlin.

- 2000b. Astronominnen in Berlin und Potsdam. In Dick, W. R. \& Fritze, K. (eds). 300 Jahre Astronomie in Berlin und Potsdam. pp. 121-141, Harri Deutsch, Frankfurt/M.

- 2000c. Women in Army Research: Ambivalent Careers in Nazi Germany. In Oldenziel, R. Camnel, A., Zachmann, K. (eds). Crossing Boundaries Building Bridges: Comparing the History of Women Engineers. 1870s1990. pp. 189-209, Harwood Academica Publications. Amsterdam.

- 2001. Der lange Weg zur Gleichberechtigung? Zur Geschichte der Habilitation von Frauen an deutschen Universitäten. - Zeitschrift für Frauenforschung \& $\mathrm{Ge}$ schlechterstudien 19 (4): 85-94.

Wegel, I. 1993. Eingriff-Umbruch-Neuaufbau. In 100 Jahre Mädchen-Gymnasium in Deutschland. p. 25-43, Braun'sche Verlagsbuchhandlung, Karlsruhe.

Wiszwianski, H. 1906. Die Faktoren der Wüstenbildung. Veröffentlichungen des Instituts für Meereskunde und des Geographischen Instituts an der Universität Berlin 9: $1-90$.

Wolf, M. 1930. Alter und Entstehung des Wald-Erbacher Roteisensteins (Grube Braut im Hunstück). - Abhand- 
lungen der Preußischen geologischen Landesanstalt, Neue Folge 123: $1-105$.

Wolfe. C. J. 1999. Number of women faculty in the geosciences increasing, but slowly. - Eos 80 (12): 133-136.

Zeil, L. 1989. Frauen in der Akademie der Wissenschaften (1700-1945). In Informationen des Wissenschaftlichen Rates: Die Frau in der sozialistischen Gesellschaft. Heft 6: $57-72$.

\section{Appendix}

\section{Data of 17 women geoscientists at the Friedrich-Wilhelms-University 1906-1945, Berlin}

Helene Wiszwianski (16. 12. 1881 Neuchâtel. Switzerland-?) Date of Dissertation: 19.5. 1906. Subjects of study: Geography; geology, meteorologie. philosophy. Title of thesis: Die Faktoren der Wüstenbildung (Factors causing desert development).

Published: Wiszwianski (1906) - s. Literature.

Advisers: E. D. von Drygalski, W. K. v. Branco (since 1907: Branca)

Parents: Mag. pharm. Samuel Wiszwianski and Sophie, neé Tugendhold

Source: Archive HUB: Phil. Fak. Nr. 412, Bl. 209-249.

Antonie Täuber, Antonie Täuber-Stremme (31. 1. 1882 Berlin-4. 8.1961 Berlin)

Date of Dissertation: 28.6.1913. Subjects of study: Geology; mineralogy, geography, philosophy. Title of thesis: Lage und Beziehungen einiger tertiärer Vulkangebiete Mitteleuropas zu gleichzeitigen Meeren oder großen Seen (Setting and relationship of some middle European Tertiary volcanic regions to coeval oceans and large lakes).

Published: Täuber (1913) - s. Literature/won a faculty prize Advisers: W. K. v. Branca, A. Penck

Parents: Royal railway-engineer Erhard Täuber and Rosalie, neé Lewin.

1912 married to Hermann Stremme (1879-1961), associate professor (Privatdozent) at the FWU Berlin 1909-1914, then senior professor at Danzig. from 1946 Berlin-East. Children: 1 daughter, 1 son (also geologist).

Sources: Archive HUB: Phil. Fak. Nr. 534, Bl. 137-193. Dr. Stremme, pers. interview with the senior author $(2000)$ and a letter including documents. curriculum vitae and photographs (2002), Dr. Ullrich Wutzke (Tel. July 2000).

Dorothea Mesch (7. 12, 1890 Berlin-?)

Date of Dissertation: 24.6.1914. Subjects of study: Mineralogy; physics, chemistry, philosophy. Title of thesis: Die Basalte des Kamerungebirges und des Gebietes zwischen Kamerungebirge und Elefantensee (The basalts of the Cameroon mountains and areas between the Cameroon mountains and Elephant Lake).

Advisers: T. Liebisch, W. K. v. Branca.

Parents: Lawyer Emil Mesch and Therese. neé Koehler. Source: Archive HUB: Phil. Fak. Nr. 550, Bl. 232-284.

Elfriede Gerwien (07. 02. 1881 in Wesel, Rheinprovinz-?) Date of Dissertation: 5.8.1914. Subjects of study: Geography; chemistry, geology, philosophy. Title of thesis: Der Lauf der Oberweser im Buntsandsteingewölbe (The course of the upper Weser in the Buntsandstein-Formations).

Advisers: A. Penck, W. K. v. Branca.

Father: (Retired) Colonel (Oberstleutnant a. D.) Paul Gerwien.

Source: Archive HUB: Phil. Fak. Nr. 553, Bl. 280-334.

Charlotte Möller (= Lotte Möller) (17.6.1893 Koblenz 22. 6. 1973 in Göttingen)

Date of Dissertation: 23.2. 1925.

Subjects of study: Geography; geology, physics, philosophy.
Title of thesis: Die Deviation bei Strommessungen im Meere (The variability of current measurements in the Ocean).

Advisers: A. Merz, A. Penck.

Father: Senior accountant (Rechnungsrat) in the Ministery of Agriculture

1925 until 1935 assistant professor at the Institute and $\mathrm{Mu}$ seum of Oceanography (Institut und Museum für Meereskunde), later Curator. Habilitation: 11.7.1929 for the subject geography/hydrology. 1929 associate professor (Privatdozentin), 1935 employed as professor, 1939-1945 full professor at the Berlin University. 25. 5. 1940 Member of the Leopoldina. Since 1952 teaching position at the Göttingen University. 1956 professorship at the Göttingen University

Sources: Archive HUB, Phil. Fak. Nr. 629, Bl. 158-165 (Prom.). Archive HUB: Phil. Fak. Nr. 1243, Bl. 367-383 (Habil. 1929)

Literature: Boedeker (1974: 55), Vogt (1996), Brosin (1999).

Hertha Sieverts-Doreck (15.7.1899 Völklingen near Saarbrücken-30. 3.1991 Stuttgart-Degerloch)

Date of Dissertation 2.3.1928. Subjects of study: Geology; zoology, mineralogy, philosophy. Title of thesis: Über die Crinoidengattung Marsupites (On the crinoid genus Marsupites). Advisers: J. F. Pompeckj, K. Zimmer.

Father: Director of a factory (Fabrikdirektor) Wilhelm Georg Sieverts.

1930-1936 at the TH Berlin, TH Karlsruhe, training at the Prussian Geological Survey to be a field geologist (Landesgeologin). 1936 married to W. Doreck and moved to Stuttgart. Children: 2 daughters. Activities: Many publications on palaeontological themes, mainly crinoids.

Sources: Archive HUB: Phil. Fak. Nr. 667, Bl. 166-176. Literature: Orbituary (Haude 1991)

Gertrud Bobek, neé Denner (15.11.1898 Bingen am Rhein-25. 6. 2000 in Taucha)

Date of Dissertation: 22.5.1928. Subjects of study: Geography: geology, botany, philosophy. Title of thesis: Die Ödländereien Deutschlands - ihre Lage und der Stand ihrer Kultivierung (The wastelands of Germany - their location and the status of their cultivation).

Advisers: A. Penck, A. Rühl.

Father: Senior civil engineer (Oberbaurat) Otto Denner, teacher at the Technical College at Nuremberg.

Since 1925/26 secretary in Berlin at the "Society for Geography (Gesellschaft für Erdkunde)". 1926 married to the physiological chemist Dr. Felix Bobek of German-Austrian nationality. Children: 2 daughters. 1933-1935 F. Bobek involved in oppositional and illegal work against the Nazis and for the German Communist Party (KPD). 1935 F. Bobek is imprisoned. 1937 death sentence. 22.1.1938 execution (Bobek 1974, S. 433). April 1935 until May 1945 emigration together with her 2 daughters to the Soviet Union, worked first as a secretary and translator at the International Agricultural Institute in Moscow, later as a scientist at the Geographic Institute of Academy of Sciences in Moscow (Bobek 1974: 432). 28.5. 1945 back in Germany, in the Eastern part of the country $=\mathrm{SBZ}$, later German Democratic Republic (GDR), there political work, worked as educator, later Party politician (Staatsfunktionärin).

Sources: Archive HUB: Phil. Fak. Nr. 668, BI. 104-119.

Literature: Bobek (1974 and 1998).

Gertrud Kobe (15. 5. 1905 Berlin - 17. 5.1995 Berlin)

Date of Dissertation: 17.10.1934. Subjects of study: Geophysics (oceanography, meteorology); geography, philosophy. Title of thesis: Der hydrographische Aufbau und die dadurch bedingten Strömungen im Skagerrak (The hydrographic setting and resultant currents in the Skagerrak). Advisers: J. M. Defant, H. von Ficker. Father: Senior policeman (Polizei-Obermeister) Paul Kobe. Until 1928 worked as accountant, mostly at Siemens \& Halske, later studies. 1934-1935 student assistant at the Institute and Museum of Oceanography. 1935-1938 Assis- 
tant at the Meteorological Institute, Berlin University. Aug. 1938-Oct. 1945 Scientist (Referentin), later group leader, at the Wilhelmshafen Marine Observatory, later at Greifswald. Oct. 1945-1. 10.1965 (Emer.) Humboldt University. 19481962 acting Director of the Meteorological Institute. Sources: Archive HUB, Phil. Fak. Nr. 764, Bl. 18-34. Orbituary (Hannelore Bernhardt) in: Humboldt. Die Zeitung der Alma Mater Berolinensis, Jg. 39, 6. 7. 1995: 2 (with several mistakes).

Literature: Vogt (2000b: 136-141), Vogt (2000c: 195-198).

Hilde Wohlgemuth, neé Heimann (9.12.1909 in WuppertalElberfeld-)

Date of Dissertation: 16. 10. 1935. Subjects of study: Geography/geology; mineralogy, philosophy. Title of thesis: Konya. Geographie einer Oasenstadt (Konya, geography of an oasis town), dedicated to her parents

Advisers: N. Krebs, W. Vogel.

Parents: Merchant Bernhard Heimann and Claire, neé Levy. Lives in Stockholm. Sweden.

Activities: Published on Jewish history (s. Literature)

Source: Archiv HUB: Phil. Fak. Nr. 798, B1. 27-48b.

Kläre Masuch (29.11. 1901 in Plaussen, Eastern Prussia-?) Date of Dissertation: 5.2.1936. Subjects of study: Geography; meteorology, physics, philosophy. Title of thesis: Zur Frage der Talmäander (On the question of river meanders). Advisers: N. Krebs, A. Rühl.

Parents: Master Blacksmith Karl Masuch and Katharina, neé Erdmann.

Later employee at the Atlas-Company der Preußischen Akademie der Wissenschaften.

Sources: Archiv HUB: Phil. Fak. Nr. 806, Bl. 1-15.

Literature: Liane Zeil (1989), S. 68 (there: Klara Masuch).

Information: Frau Petra Hoffmann (13. 1. 1999).

Ferdinande Michael (dates unknown)

Studies at the Technische Hochschule (TH) Berlin?

Adviser: W. Gothan.

Publication: Michael (1936).

Irma Iwan-Schaberow (6. 1. 1902 Berlin-?)

Date of Dissertation: 10.12.1936. Subjects of study: Geography; geology, physics. Title of thesis: Beiträge zur Morphologie des Oberpfälzer Waldes (Contributions to the morphology of the Upper Pfalz Hills).

Advisers: N. Krebs, F. E. A. Solger.

Parents: Merchant Gustav Schaberow and Margarete, neé Buntebardt.

Source: Archiv HUB: Phil. Fak. Nr. 834, Bl. 59-90.

Marie-Luise Teichmuiller (Marlis Teichmüller), neé Köster (11. 11. 1914 Herne - 12.09. 2000 Duisburg)

Dissertation: 31.3.1941. Subjects of study: Geology; mineralogie, geography. Title of thesis: Der Feinbau amerikanischer Kohlen im Anschliff und Dünnschliff. Ein Vergleich kohlenpetrographischer Untersuchungsmethoden (The fine structure of American coals in polished section and thin section. A comparison of coal petrographic analysis methods).

Published: Teichmüller (1941).

Advisers: E. Stach, H. Stille.

Parents: Dipl. Ing. Emil Köster and Klara, neé Böcker.

July 1938 married with the geologist and assistant professor Dr. habil. Rolf Teichmüller. 1941-1945 Geological Survey of Germany (Geologisches Reichsamt) Berlin. 1947-1979 Geological Survey of the State of Nordrhein-Westfalen (Geolo- gisches Landesamt des Landes Nordrhein-Westfalen) in Krefeld, leader of a working group, director.

Sources: Math.-Nat. Fak. Nr. 166, Bl.1-20. Letters, curriculum vitae and list of publications to the senior author (March 1998, Febr. 2000); letters from Mrs. Dr. I. Hirdes about childhood and youth (2000 and May 2003).

Literature: Biographies in: TSOP Newsletter, Vol. 11 (3-4), p. 15, 1994 and Kasig (1983).

Erna Hein (9.8.1902 Berlin-?)

Date of Dissertation: 15.1.1943. Subjects of study: Geophysics, geography; chemistry. Title of thesis: Hydrographische Untersuchung des Heinitzsees in Rüdersdorf/Mark (Hydrological studies on the Lake Heinitz near Rüdersdorf/Mark). Advisers: Lotte Möller, N. Krebs.

Parents: Railway employee (Eisenbahnbeamter) Gustav Hein und Emilie, neé Krüger.

since 1934 teacher in public schools, on the side studies of geography.

Source: Archive HUB: Math.-Nat. Fak. Nr. 183, Bl. 1-19.

Ruth Rabien, geb. Ballhorn (5. 5. 1915 Basel, Switzerland-?) Date of Dissertation: 4.6.1943. Subjects of study: Geography, geology; prehistory. Title of thesis: Beiträge zur Morphologie der Veluwe (Holland), mit Karten. (Contributions to the morphology of the Veluwe Hills (Holland), with maps). Advisers: H. Lehmann, N. Krebs.

Parents: Member of Foreign Office staff (Ministerialamtmann im Auswärtigen Amt) Oskar Ballhorn und Ehefrau Anna, neé Jenssen.

Since 1942 married to Hans-Richard Rabien (born 1916). Source: Archive HUB: Math.-Nat. Fak. Nr. 187, Bl. 52-66.

Barbara Wallstabe (05. 02.1915 Magdeburg-?)

Date of Dissertation: 19.10.1944. Subjects of study: Geography; meteorology. Title of thesis: Über den Stand der kartographischen Darstellung der Erde um 1943 (on the status of cartographic presentation of the earth as of 1943).

Advisers: N. Krebs, Lotte Möller.

Parents: High school Principal (Oberstudiendirektor) Dr. Friedrich Wallstabe and Hanna, neé Knaut.

Febr. 1941-1.2.1944: scientific assistant at the Geographic Institute. 1.7.1944-1945: scientific assistant at the Institute of Oceanography of the Berlin University.

Source: Archive HUB: Math.-Nat. Fak. Nr. 199, Bl. 1-16.

Ingeborg Böwing, neé Bathke (9. 9.1911 Berlin-)

Promotion: 5. 3. 1945. Subjects of study: Palaeobotany, pharmaceutical chemistry; botany. Title of thesis: Das Alter der samländischen und ostpommerschen Braunkohlenformation auf Grund der Flora (The ages of the Samlandian and EastPommeranian brown coal formations based on the flora).

Advisers: W. Gothan, L. Diels.

Parents: Primary school teacher (Volksschullehrer) Eduard Bathke and Johanna, neé Schwabe.

Since 1934 married to the pharmacist and owner of a pharmacy Hans Böwing (1894-1945), 4 children, afterwards studies of pharmacy. 1945: at the Central Government Geological Survey (Reichsamt für Bodenforschung) Berlin. After the sudden death of her husband in 1945 she took over the family owned pharmacy, 1945-1951 in the Friedrichstraße in Berlin-Mitte, since 1951 in Berlin-Grunewald. Lives today in Kleinmachnow near Berlin.

Sources: Archive HUB: Math.-Nat. Fak. Nr. 206, BI. 15-27. Interview with the authors in Nov. 1998 in Berlin-Zehlendorf. 